
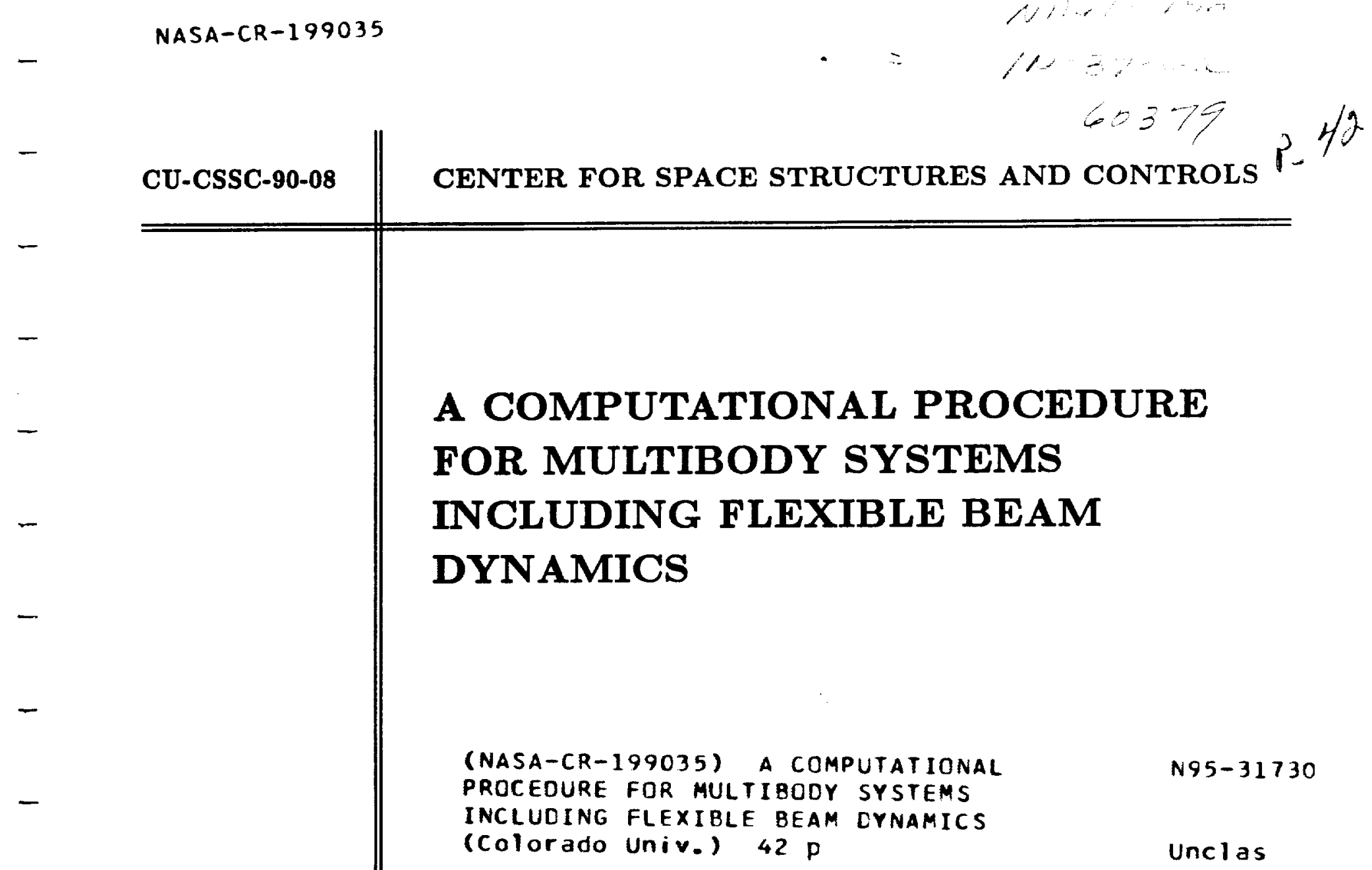

by

J. D. Downer, K. C. Park, and J. C. Chiou

COLLEGE OF ENGINEERING UNIVERSITY OF COLORADO

CAMPUS BOX 429

BOULDER, COLORADO 80309 


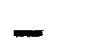

$-$

$-$

$-$

$-$

-

$-$

$-$

-

$-$

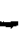

$-$

-

-

$-$

$-$

$-$

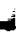

,

2 


\title{
A COMPUTATIONAL PROCEDURE FOR MULTIBODY SYSTEMS INCLUDING FLEXIBLE BEAM DYNAMICS
}

\author{
J. D. Downer, K. C. Park, and J. C. Chiou \\ Department of Aerospace Engineering Sciences and \\ Center for Space Structures and Controls \\ University of Colorado, Campus Box 429 \\ Boulder, Colorado 80309
}

April 1990

Report No. CU-CSSC-90-08 


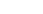




\title{
A Computational Procedure for Multibody
} Systems Including Flexible Beam Dynamics

J. D. Downer, K. C. Park, and J. C. Chiou

Department of Aerospace Engineering Sciences

and Center for Space Structures and Controls

University of Colorado at Boulder

Boulder, CO 80309-0429, USA

\begin{abstract}
A computational procedure suitable for the solution of equations of motions for flexible multibody systems has been developed. The flexible beams are modeled using a fully nonlinear theory which accounts for both finite rotations and large deformations. The present formulation incorporates physical measures of conjugate Cauchy stress and covariant strain increments. As a consequence, the beam model can easily be interfaced with real-time strain measurements and feedback control systems. A distinct feature of the present work is the computational preservation of total energy for undamped systems; this is obtained via an objective strain increment/stress update procedure combined with an energy-conserving time integration algorithm which contains an accurate update of angular orientations. The procedure is demonstrated via several example problems.
\end{abstract}

\section{Introduction}

The simulation of flexible multibody systems is becoming an increasingly important tool for the design and operation of many engineering applications. Typical examples of such systems include deployable space structures, high precision machine dynamics and robotics, and other problems containing controlled positioning of structural components. The components of these articulated structures typically undergo large relative displacements and rotations in order to carry out the intended operations. To perform the desired kinematic motions, various types of mechanical joints are introduced to constrain the relative motion between the various components. New technology needs of both the space and robotics industries have increased the demand for accurate numerical simulations of the effect of component flexibility on the performance of multibody systems. A significant coupling between the gross structural motion and the elastic deformation can be experienced by typical applications in which lightweight structures with higher flexibility are required to operate with greater positioning accuracy and at higher speeds. To capture this phenomenon, a realistic mathematical model of the structural component that can readily be incorporated into a general multibody dynamics methodology is necessary.

Two basic approaches, the floating frame approach and the nonlinear continuum approach, exist for the modeling of flexible components within a general multibody system. 
The floating frame approach introduces a moving reference frame to follow some overall mean rigid body motion of the beam; the elastic deformation of the beam is then described relative to this moving reference ${ }^{1-6}$. With this approach, the classical multi-rigid body analysis was extended to include structural flexibility by superposing existing linear deformation descriptions onto the rigid motions of the floating reference frame ${ }^{7,8}$. The definition of such a mean axis system and the corresponding deformation modes within the general context of the finite element method has been presented ${ }^{9-11}$. To minimize the number of elastic coordinates, coordinate transformations from the physical elastic coordinates to modal coordinates were performed within the multibody dynamics context ${ }^{12}$, and static correction modes were used in conjunction with the normal modes of vibration to account for reaction forces and torques transmitted to the components through joint connections $^{13,14}$. An alternative choice of a floating reference frame for finite element applications, termed the convected coordinate system, was introduced as a simple separation of the rigid body motion and the structural deformation for a given finite element ${ }^{15-18}$. All of these studies, however, are limited by the assumption of linear deformation theory which is inadequate to capture certain nonlinear phenomena. Nonlinear deformation theories must be taken into account for such instances as the geometric stiffening of a spinning beam ${ }^{19,20}$ in which the structural component experiences a centrifugal force as well as applications in which the components necessarily have low mass and very high flexibility. Extensions of the original approach to model the nonlinear effects include the substructuring technique in which the component is further partitioned into substructures each with a local reference frame where normal vibration and static correction modes can then be used to model the deformation ${ }^{21}$, and the finite element incorporation of a nonlinear Green strain measure ${ }^{22,23}$. The resulting equations of motion of the floating frame approach, written in terms of a set of reference coordinates and a set of relative elastic coordinates, inherently contain a complex coupling of the gross motion and the elastic deformation modes.

Recently, a fully nonlinear continuum approach to describe the dynamics of the flexible beam has been pursued ${ }^{24-28}$. Through the use of finite-deformation rod theories ${ }^{29-32}$, the approach is capable of directly accounting for both finite rotation kinematics and large deformations of the beam component. Since the motion due to rigid rotations of the beam is not distinguished from that due to deformations, the need for a floating reference frame is completely obviated and the component inertia is identical in form to that of a rigid body. The inherent nonlinear coupling between the gross body motion and the elastic deformation is transferred to the stiffness part of the equations of motion. The key to the successful adoption of this approach is to develop a computational procedure for the nonlinear internal force term that preserves rigid body motions.

The aim of this paper is to incorporate the nonlinear continuum formulation of the spatial beam motion into a general multibody dynamics software methodology. The present formulation employs a convected coordinate representation of physical Cauchy stresses and corresponding set of physical strains. This representation naturally lends itself to the "software in the real-time experiment" loop as sensors measure only physical quantities. 
Another advantage of the formulation is that the degrees of freedom of the beam component embody both the rigid and flexible deformation motions. The task for incorporating the multibody system constraints becomes straightforward, and the equations of motion for an arbitrary configuration of flexible beams and rigid bodies can automatically be generated in terms of an identical set of physical coordinates. Numerical solution procedures for the integration of spatial kinematic systems can then be directly applied to these physical coordinates. Such a universal treatment is not applicable within the context of the floating frame approach as the reference and elastic coordinate definitions are of highly different character.

The rest of the paper will be organized as follows. Section 2 will detail the kinematic description of the continuum beam in which the total motion is referred directly to the inertial reference frame. The principle of virtual work of a continuum as specialized to the spatial motion of the beam component is detailed in Section 3. The subsequent finite element discretization of the beam component and overall multibody system equations are then presented. Section 4 will summarize the staggered procedure for the integration of multibody dynamic systems. The virtual work expression is used to derive the method of computation of the internal force, and Section 5 will address this algorithmic treatment of the nonlinear stiffness operator. Section 6 will present some example problems demonstrating the software capabilities.

\section{Beam Kinematics}

The present formulation adopts an inertial reference frame for describing the translational motions and a body-fixed frame for the rotational motions. The consequence of this description is that the translational and rotational variables embody information due to both rigid rotations and deformations of the beam. The configuration of the beam, as shown in Figure 1, is completely characterized using a position vector locating the neutral axis of the beam from the inertial origin and a body-fixed frame representing the orientation of the cross-section with respect to the inertial reference frame. The position vector $r$ locating an arbitrary particle point on the beam is thus described as

$$
\mathbf{r}=(X+u)^{T} \mathbf{e}+\ell^{T} \mathbf{b}
$$

where "boldface" symbols represent three subscripted vectors and the normal type symbols represent three components of a given vector; $\mathbf{e}=\left\{\mathbf{e}_{1}, \mathbf{e}_{2}, \mathbf{e}_{\mathbf{3}}\right\}^{T}$ represents the three orthogonal vectors defining the inertial reference frame; $\mathbf{b}=\left\{\mathbf{b}_{\mathbf{1}}, \mathbf{b}_{\mathbf{2}}, \mathbf{b}_{\mathbf{3}}\right\}^{T}$ represents the body-fixed reference frame which is attached to and rotates with the beam cross section; $X=\left\{X_{1}, X_{2}, X_{3}\right\}^{T}$ represents the inertial components of the original neutral axis position; $u=\left\{u_{1}, u_{2}, u_{3}\right\}^{T}$ represents the inertial components of the subsequent total translational displacement of the neutral axis, and $\ell^{T}=\left\{0, \ell_{2}, \ell_{3}\right\}$ are the body-fixed components of the distance from the beam neutral-axis to the material point located on the deformed beam cross-section. It is noted that the beam cross-section is allowed to 
rotate such that it is not necessarily perpendicular to the neutral axis in order to model transverse shear deformations. Warping deformation of the cross-section is not taken into consideration.

In order to derive the necessary time derivatives for the description of the large rotation dynamics, we employ the well known formula ${ }^{33}$ :

$$
\frac{d}{d t} \equiv \frac{d^{e}}{d t}=\frac{d^{b}}{d t}+\omega \times
$$

where $\boldsymbol{\omega}$ is the angular velocity vector and the superscripts $e$ and $b$ indicate that the derivatives are to be those observed in the inertial (space) and body (rotating) system of axes respectively. The above is expressed in the matrix form to act on the body frame components of a given vector

$$
\frac{d}{d t}=\frac{d^{b}}{d t}+\tilde{\omega}
$$

and the velocity and acceleration of the position vector (2.1) are

$$
\begin{aligned}
\frac{d \mathbf{r}}{d t} & =\frac{d u^{T}}{d t} \mathbf{e}+\ell^{T} \tilde{\omega}^{T} \mathbf{b} \\
\frac{d^{2} \mathbf{r}}{d t^{2}} & =\frac{d^{2} u^{T}}{d t^{2}} \mathbf{e}+\ell^{T}\left(\frac{d^{b} \tilde{\omega}^{T}}{d t}+\tilde{\omega}^{T} \tilde{\omega}^{T}\right) \mathbf{b} .
\end{aligned}
$$

Given the following relation between the b-basis and the e-basis

$$
\mathbf{b}=\mathbf{R} \mathbf{e}
$$

where $\mathbf{R}$ is a $(3 \times 3)$ orthogonal transformation matrix, the body frame components of the skew-symmetric angular velocity tensor $\left(\tilde{\omega}^{T}\right)$ are

$$
\tilde{\omega}^{T}=\frac{d \mathbf{R}}{d t} \mathbf{R}^{T}
$$

A conjugate virtual rotation tensor is defined analogous to the above as

$$
\delta \tilde{\alpha}^{T}=\delta \mathbf{R R}^{T},
$$

and the variation of the position vector (2.1) is given as

$$
\delta \mathbf{r}=\delta u^{T} \mathbf{e}+\ell^{T} \delta \tilde{\alpha}^{T} \mathbf{b}
$$

The equations of motion as derived from the stated beam kinematic description will be discussed next. 


\section{Spatial Beam Equations of Motion}

The principle of virtual work, which is simply a 'weak' or variational form of Cauchy's differential equations of motion for the equilibrium of a given set of particles of a continuum, is stated as $^{34}$

$$
\int_{V} \delta r_{i} \rho \ddot{r}_{i} d V+\int_{V} \sigma_{i j}^{e} \frac{\partial \delta r_{i}}{\partial x_{j}} d V=\int_{V} \delta r_{i} f_{i} d V+\int_{S} \delta r_{i} t_{i} d S .
$$

The cartesian coordinates $x_{i}$ represent the particle position after some deformation has taken place, $\delta r_{i}$ a kinematically admissible virtual displacement, $\ddot{r}_{i}$ the acceleration, $f_{i}$ the external force per unit mass, and $t_{i}$ the stress vector acting on a surface with outward normal components $n_{i}$. Likewise, $\sigma_{i j}^{e}$ represents the cartesian components of the Cauchy stress tensor, and $\rho$ is the mass density. The expression is tailored for the continuum beam by using the kinematic relations $(2.1),(2.4)$, and (2.8) for the components $x_{i}, \delta r_{i}$, and $\ddot{r}_{i}$ respectively. As well as providing the basis for a finite element approximation techniques, the variational formulation readily lends itself to the derivation of incremental strain-displacement relations as deduced from the derivatives of the virtual displacement components. The present formulation employs a physical stress measure defined as a force per unit deformed area and the conjugate physical strain increments based on the deformed coordinates. As such, the formulation can be recast into a convected coordinate system moving with the beam, thus simplifying the stress and strain computational procedures. The practical advantages of such a formalism are in real-time software simulation experiments as the computed physical quantities correspond to the actual stress/strain measurements of the sensors located and operating on the deformed structure.

For notational convenience and subsequent finite element discretization, the principle of virtual work is expressed in the following operator form:

$$
\delta F^{I}+\delta F^{S}=\delta F^{E}+\delta F^{T}
$$

where the inertia operator $\delta F^{I}$, internal force operator $\delta F^{S}$, external force operator $\delta F^{E}$. and traction operator $\delta F^{T}$ are identified from (3.1). Explicit expressions for the various operators incorporating the large rotation beam kinematics are derived in Sections 3.1 to 3.3. The finite element discretizations are given in Section 3.4, and the incorporation of the beam formulation into the multibody dynamics framework is discussed in Section 3.5.

\subsection{Spatial Beam Inertia Operator}

The inertia operator was defined from (3.1) as

$$
\delta F^{I}=\int_{V} \rho \delta r_{i} \ddot{r}_{i} d V=\int_{V} \rho \delta \mathbf{r} \cdot \ddot{\mathbf{r}} d V
$$


from which an expression can be derived directly from the kinematic equations (2.4) and (2.8). If the origin of the body-fixed basis is located at the centroid of the cross-section, the following simple expression results for $\delta F^{I}$ :

$$
\delta F^{I}=\int_{s}\left\{\delta u^{T} \delta \alpha^{T}\right\}\left\{\begin{array}{c}
\rho A \frac{d^{2} u}{d t^{2}} \\
J \frac{d^{6} \omega}{d t}+\tilde{\omega} J \omega
\end{array}\right\} d s
$$

where

$$
\int_{A} \rho \tilde{\ell} \tilde{\ell}^{T} d A=\mathbf{J}
$$

represents the inertia tensor of the beam cross-section and $d s$ represents the remaining integration to be performed over a beam length parameter. The translational inertia is completely decoupled from the rotary inertia and is of the same form as that seen in rigid body dynamics. This is due to the dual choice of the translational displacements measured in the inertial basis and the angular velocity measured in the body-fixed basis located at the center of mass of the cross-section.

\subsection{Spatial Beam Internal Force Operator}

The internal force operator was defined in (3.1) as

$$
\delta \mathbf{F}^{\mathbf{S}}=\int_{V} \frac{\partial \delta r_{i}}{\partial x_{j}} \sigma_{i j}^{e} d V
$$

identifying as conjugate quantities the virtual displacement gradient and the Cauchy stress tensor. This form of the internal force along with the beam kinematic description will be used to deduce a set of virtual strain-displacement relations that are invariant to rigid body motions. The corresponding conjugate stress tensor will be obtained from an objective incremental procedure that relates incremental strains obtained from the virtual strain tensor to Cauchy stress increments. Thus the internal force term will be derived completely from the original definition of the beam kinematics without making an a priori definition of the existing strains or stresses.

A physically appealing decomposition of the stress and virtual strain tensors into an alternative beam reference frame which lies tangent to the deformed neutral axis is introduced to provide conceptual simplifications in the derivation and subsequent computations. Certain stress states referenced to this convected frame are kinematically required to vanish in a beam formulation. When applied to the convected frame stress components, this choice also leads the task of stress update computations to a simple additive procedure. To this end, we introduce a convected reference frame, denoted by a, which is related to the inertial reference frame e by

$$
\mathbf{a}=\mathbf{T} \mathbf{e}, \quad \mathbf{a}=\left\{\mathbf{a}_{1}, \mathbf{a}_{2}, \mathbf{a}_{3}\right\}^{T} .
$$


For implementation purposes within the context of the finite element method, the convected frame will be constant on the element level and thus is similar in concept to that introduced by Belytschko et al. ${ }^{15,16}$. It is noted that this reference frame does not coincide with the body frame $b$ attached to the cross-section. The relative difference between these two reference frames is represented by the rotation matrix $\mathbf{S}$ which models the effects of transverse shear and torsion deformation as

$$
\mathbf{b}=\mathbf{S} \mathbf{a}, \quad \mathbf{R}=\mathbf{S} \mathbf{T},
$$

and the latter interdependence between the rotation matrices is established.

The internal force operator, originally characterized by the inertial frame components of the Cauchy stress tensor ( $\left.\sigma_{i j}^{e}\right)$ and conjugate virtual displacement gradient, will equivalently be expressed in terms of the convected frame components of the stress tensor $\left(\sigma_{i j}^{a}\right)$ and a corresponding convected virtual displacement gradient as

$$
\delta F^{S} \equiv \int_{V} \frac{\partial \delta r_{i}}{\partial x_{j}} \sigma_{i j}^{e} d V=\int_{V} T_{m i} \frac{\partial \delta r_{i}}{\partial \xi_{k}} \sigma_{m k}^{a} d V .
$$

The symmetric portion of the transformed deformation gradient is used to define the virtual strain tensor $\delta \varepsilon_{m k}^{a}$ as

$$
\delta \varepsilon_{m k}^{a} \equiv \frac{1}{2}\left(T_{m i} \frac{\partial \delta r_{i}}{\partial \xi_{k}}+T_{k i} \frac{\partial \delta r_{i}}{\partial \xi_{m}}\right)
$$

which is an objective tensor invariant to arbitrary rigid body motions. The internal force, written in terms of the convected frame tensors, will be expressed in vector format as

$$
\delta F^{S}=\int_{V} \delta \varepsilon_{m k}^{a} \sigma_{m k}^{a} d V=\int_{V}\left\{\begin{array}{lll}
\sigma_{\xi \xi} & \widehat{\sigma}_{\xi \eta} & \widehat{\sigma}_{\xi \zeta}
\end{array}\right\}\left\{\begin{array}{l}
\delta \varepsilon_{\xi \xi} \\
\delta \widehat{\varepsilon}_{\xi \eta} \\
\delta \widehat{\varepsilon}_{\xi \zeta}
\end{array}\right\} d V
$$

where the notation

$$
\xi_{i}=\left\{\xi_{1}, \xi_{2}, \xi_{3}\right\}=\{\xi, \eta, \zeta\}, \quad()_{i j}=()_{i j}+()_{j i}
$$

denotes the coordinates of the convected reference frame and the engineering shear strain definitions respectively. The rest of the convected frame strain components

$$
\delta \varepsilon_{\eta \eta}, \delta \varepsilon_{\zeta \zeta}, \delta \widehat{\varepsilon}_{\eta \zeta}
$$

are identically equal to zero due to the original assumptions of the beam kinematics.

A set of virtual strain-displacement relations can be derived from the expressions (2.8) and (3.9). The final result is expressed as

$$
\left\{\begin{array}{l}
\delta \varepsilon_{\xi \xi} \\
\delta \widehat{\varepsilon}_{\xi \eta} \\
\delta \widehat{\varepsilon}_{\xi \zeta}
\end{array}\right\}=\delta \gamma+\tilde{\ell}^{T} \delta \kappa
$$


where

$$
\delta \gamma=\mathbf{T} \frac{\partial \delta u}{\partial \xi}+\left\{\begin{array}{c}
0 \\
-\delta \beta_{3} \\
\delta \beta_{2}
\end{array}\right\}, \quad \delta \kappa=\frac{\partial \delta \beta}{\partial \xi}, \quad \delta \beta=\mathbf{S}^{T} \delta \alpha
$$

and is comparable to that of Reissner ${ }^{31}$. In the above expressions, $\delta \gamma$ represents the membrane and two transverse shear strains, $\delta \kappa$ the torsion and two bending strains, and $\delta \beta$ the virtual rotations of the cross-section referred to the convected frame.

In an analogous manner the total stress state is expressed as

$$
\left\{\begin{array}{l}
\sigma_{\xi \xi} \\
\sigma_{\xi \eta} \\
\sigma_{\xi \zeta}
\end{array}\right\}=\sigma_{\gamma}+\tilde{\ell}^{T} \sigma_{\kappa}
$$

to be obtained from a separate stress update procedure. A substitution of (3.11) and (3.13) into (3.10), and a spatial integration over a symmetric cross-sectional area results in the following expression for the internal force

$$
\delta F^{S}=\int_{\xi}\left\{\delta \gamma^{T} N_{\gamma}+\delta \kappa^{T} M_{\kappa}\right\} d \xi
$$

where $N_{\gamma}$ represent the axial and transverse shear forces per unit length, and $M_{\kappa}$ represent the torsional and bending moments per unit length as given by

$$
N_{\gamma}=\int_{A} \sigma d A, \quad M_{\kappa}=\int_{A} \tilde{\ell}^{T} \sigma d A
$$

To be consistent with the inertia operator derived in (3.4), the above is written as

$$
\delta F^{S}=\int_{\xi}\left\{\begin{array}{lll}
\delta u^{T} & \left.\delta \alpha^{T}\right\} & {[B}
\end{array}\right]^{T}\left\{\begin{array}{l}
N_{\gamma} \\
M_{\kappa}
\end{array}\right\} d \xi
$$

which involves a transformation back to the body frame components of the virtual rotations and also an identification of the desired incremental strain-displacement matrix $B$. To effect the change of the body reference frame of the cross-section orientation in space with respect to the constant convected reference frame, we invoke the following relations:

$$
\begin{aligned}
\frac{\partial^{a} \delta \beta}{\partial \xi}=S^{T} \frac{\partial^{a} \delta \alpha}{\partial \xi} & =S^{T}\left(\frac{\partial^{b} \delta \alpha}{\partial \xi}+\tilde{\kappa}_{S} \delta \alpha\right) \\
\tilde{\kappa}_{S}^{T} & =\frac{\partial^{a} \mathbf{S}}{\partial \xi} \mathbf{S}^{T}
\end{aligned}
$$


which are completely analogous to those relating changes in the time derivative given in (2.3) and (2.6). The strain operator $[B]$ of (3.16) is then recognized as

$$
[B]=\left[\begin{array}{cc}
\mathrm{T} \frac{\partial^{a}}{\partial \xi} & \tilde{i}_{1} \mathbf{S}^{T} \\
\mathbf{0} & \mathbf{S}^{T}\left(\tilde{\kappa}_{S}+\mathbf{I} \frac{\partial^{b}}{\partial \xi}\right)
\end{array}\right], \quad \tilde{i}_{1}=\left[\begin{array}{ccc}
0 & 0 & 0 \\
0 & 0 & -1 \\
0 & 1 & 0
\end{array}\right]
$$

It remains to provide a procedure for updating $\sigma_{\gamma}$ and $\sigma_{\kappa}$ in order to compute $N_{\gamma}$ and $M_{\kappa}$. For this purpose, we employ the following rate-type law that relates the instantaneous rate of stress to the instantaneous rate of deformation:

$$
\dot{\sigma}_{k l}^{a} \simeq c_{k l m p} \dot{\varepsilon}_{m p}^{a}
$$

where $c_{k l m p}$ represents the material response tensor, and $\dot{\sigma}_{k l}^{a}$ and $\dot{\varepsilon}_{m p}^{a}$ represent the convected frame stress and strain rates, respectively. This approximate constitutive law can be derived by transforming the Truesdell rate equation ${ }^{35}$, which is an objective equation based on inertial components of Cauchy stresses and the velocity gradient tensor, to the convected basis. This equation is then integrated in time as

$$
\begin{aligned}
\sigma_{k l}^{a}{ }^{n+1} & =\sigma_{k l}^{a}{ }^{n}+\int_{t^{n}}^{t^{n+1}} c_{k l m p} \dot{\varepsilon}_{m p}^{a} d t \\
& =\sigma_{k l}^{a}{ }^{n}+c_{k l m p} \Delta \varepsilon_{m p}^{a}
\end{aligned}
$$

to define the stress update procedure. The evaluation of the strain increments $\Delta \varepsilon_{m p}^{a}$, to be defined from the virtual strains (3.12), will be detailed in Section 5 .

\subsection{Spatial Beam External Force and Traction Operator}

The external force operator defined in (3.1) as

$$
\delta F^{E}=\int_{V} \delta r_{i} f_{i} d V
$$

has the final resultant form

$$
\delta F^{E}=\int_{\xi}\left\{\delta u^{T} \delta \alpha^{T}\right\}\left\{\begin{array}{l}
f^{e} \\
f^{b}
\end{array}\right\} d \xi
$$

where $f^{e}$ represents the inertial components of a force per unit length acting on the beam neutral axis and $f^{b}$ represents the body-fixed components of a moment per unit length acting on the beam cross-section. The traction operator defined as

$$
\delta F^{T}=\int_{S} \delta r_{i} t_{i} d S
$$


acts on the exterior surfaces of the beam as natural boundary conditions.

\subsection{Finite Element Discretization}

The variational form of the partial differential equations representing the spatial dynamics of a continuous beam presented in the preceding sections provide a basis for the finite element method to be used as a spatial discretization procedure ${ }^{36}$. In the present study, we restrict ourselves to the use of linear shape functions to approximate the displacement field along the beam, viz.,

$$
u=\sum_{I=1}^{n p e} N_{I} u_{I}
$$

where $N_{I}$ denotes the spatial linear shape functions, $u_{I}$ represents the degrees of freedom at the element nodes, and npe denotes the number of nodes per element. The element inertia operator, from (3.4), is written as

$$
\begin{aligned}
\delta F^{I}=\sum_{I=1}^{n p e} \sum_{K=1}^{n p e}\left\{\delta u_{I}^{T} \rho A M_{I K}^{E} \frac{d^{2} u_{K}}{d t^{2}}\right. & \left.+\delta \alpha_{I}^{T} \rho J_{I} M_{I K}^{E} \frac{d^{b} \omega_{K}}{d t}\right\} \\
& +\sum_{I=1}^{n p e} \delta \alpha_{I}^{T} D_{I}^{E}
\end{aligned}
$$

where

$$
M_{I K}^{E}=\int_{\xi} N_{I} N_{K} d \xi, \quad D^{E}(\omega)_{I}=\int_{\xi}(\tilde{\omega} J \omega)_{I} d \xi
$$

represent the element mass matrix and nonlinear angular acceleration vector. The former will be evaluated as a standard lumped mass matrix for the computational efficiency of explicit integration techniques to be described in Section 4, and the latter will be evaluated from an average of the element nodal angular velocities. The element internal force operator, from (3.16), is written as

$$
\delta F^{S}=\sum_{I=1}^{n p e}\left\{\begin{array}{ll}
\delta u_{I} & \delta \alpha_{I}
\end{array}\right\}\left[\begin{array}{c}
B_{I}^{E}
\end{array}\right]^{T}\left\{\begin{array}{c}
N_{\gamma} \\
M_{\kappa}
\end{array}\right\}^{E}=\sum_{I=1}^{n p e}\left\{\begin{array}{ll}
\delta u_{I} & \delta \alpha_{I}
\end{array}\right\}\left\{\begin{array}{l}
S_{I}^{e} \\
S_{I}^{b}
\end{array}\right\}^{E}
$$

where the evaluation of the element strain operator

$$
\left[B_{I}^{E}\right]=\int_{\xi}\left[\begin{array}{cc}
\mathbf{T} \frac{\partial N_{I}}{\partial \xi} & \tilde{i}_{1} N_{I} \mathbf{S}_{I}^{T} \\
0 & \mathbf{S}_{I}^{T}\left(\tilde{\kappa}_{S} N_{I}+\frac{\partial N_{I}}{\partial \xi}\right)
\end{array}\right] d \xi
$$


and the resultant element stresses $N_{\gamma}$ and $M_{\kappa}$, as defined in (3.19) and (3.15) respectively, will be presented in detail in Section 5. The element external force operator, from (3.22), is written as

$$
\delta F^{E}=\sum_{I=1}^{n p e}\left\{\begin{array}{ll}
\delta u_{I} & \delta \alpha_{I}
\end{array}\right\}\left\{\begin{array}{l}
f_{I}^{e} \\
f_{I}^{b}
\end{array}\right\}, \quad f_{I}^{e, b}=\int_{\xi} N_{I} f^{e, b} d \xi
$$

and the traction operator is implemented as boundary conditions on the nodes. The equations of motion in terms of nodal degrees of freedom $\left(\delta u_{d}, \delta \alpha_{d}\right)$ for the entire beam are obtained from an assembly of the above element operators. For the unconstrained beam, these nodal virtual displacements and rotations are arbitrary independent variations, and the discrete equations of motion are written as

$$
\left[\begin{array}{cc}
M_{d} & 0 \\
0 & J_{d}
\end{array}\right]\left\{\begin{array}{c}
\ddot{u}_{d} \\
\dot{\omega}_{d}
\end{array}\right\}+\left\{\begin{array}{c}
0 \\
D_{d}(\omega)
\end{array}\right\}+\left\{\begin{array}{c}
S_{d}^{e} \\
S_{d}^{b}
\end{array}\right\}=\left\{\begin{array}{c}
f_{d}^{e} \\
f_{d}^{b}
\end{array}\right\}
$$

where $M_{d}, J_{d}$ represent the assembled mass and inertia matrices, and $D_{d}(\omega), S_{d}, f_{d}$ represent the assembled nonlinear acceleration, internal force, and external force vectors respectively.

\subsection{Extension to Multibody Dynamics}

The present formulation of spatial beam dynamics as given by (3.29) can readily be incorporated into a general multibody dynamics methodology. The degrees of freedom of a rigid body, namely the inertially-based translational position of the center of mass and the rotational orientation of the body reference frame, coincide with the degrees of freedom of the nodal coordinates of the present beam components. Thus the equations of motion (3.29) can be specialized to represent a rigid body system by setting the internal force $S_{d}$ equal to zero.

It remains to augment both the holonomic and nonholonomic constraint conditions modeling the contacts among the various bodies to the equations of motion. For this purpose, the Lagrange multiplier technique is used to couple the algebraic constraint equations with the differential equations of motion of the generalized coordinates by augmenting the virtual work of the unconstrained system (3.2) with the virtual work required to enforce the constraints. Given a set of equations representing holonomic constraint conditions between the displacement coordinates as

$$
\Phi_{H}(u, t)=0, \quad \delta \Phi_{H}=\frac{\partial \Phi_{H}}{\partial u} \delta u=\mathbf{B}_{H} \delta u=0
$$

and a set representing nonholonomic constraint conditions between the virtual displacements and rotations as

$$
\delta \Phi_{N}(u, \delta u, R, \delta \alpha)=\mathbf{B}_{N}\left\{\begin{array}{l}
\delta u \\
\delta \alpha
\end{array}\right\}=0,
$$


the virtual work expression (3.2) of the unconstrained system is modified to account for the constraint via Lagrange's multipliers $\lambda$ as $^{37}$

$$
\delta F^{I}+\delta F^{S}+\lambda_{H} \cdot \delta \Phi_{H}+\lambda_{N} \cdot \delta \Phi_{N}=\delta F^{E}+\delta F^{T} .
$$

The virtual displacements and rotations of the generalized coordinates can now be treated as arbitrary independent variations in the modified virtual work expression. The equations of motion for constrained flexible multibody systems with respect to a set of generalized coordinates ( $\dot{u}, \omega)$ denoting both the nodal coordinates of the flexible members and the physical coordinates of the rigid bodies can be expressed as

$$
\left[\begin{array}{cc}
M & 0 \\
0 & J
\end{array}\right]\left\{\begin{array}{l}
\ddot{u} \\
\dot{\omega}
\end{array}\right\}+\mathbf{B}^{T} \lambda=\left\{\begin{array}{l}
Q_{u} \\
Q_{\omega}
\end{array}\right\}
$$

where

$$
\left\{\begin{array}{l}
Q_{u} \\
Q_{\omega}
\end{array}\right\}=\left\{\begin{array}{c}
f^{e}-S^{e}(u, q) \\
f^{b}-D(\omega)-S^{b}(u, q)
\end{array}\right\}, \quad \mathbf{B}=\left[\begin{array}{c}
B_{H} \\
B_{N}
\end{array}\right], \lambda=\left\{\begin{array}{l}
\lambda_{H} \\
\lambda_{N}
\end{array}\right\}
$$

in which $D(\omega)$ represents the nonlinear acceleration, $S$ the internal force vector, $f$ the external force vector, and $\mathbf{B}^{T} \lambda$ the constraint force vector. As an additional number of unknown Lagrange multipliers $\lambda$ for each constraint condition have been introduced along with the generalized coordinates for each degree of freedom, the above system of equations must be augmented with the constraint equations themselves to achieve a determined system of equations.

\section{Time Integration Techniques for Constrained Systems}

The present methodology to formulate the equations of motion of an arbitrary assemblage of interconnected flexible beams and rigid bodies is readily adaptable for use with existing multibody dynamics solution techniques. The equations (3.32) model the beam components with degrees of freedom $u$ and $\omega$ that embody both the rigid and flexible deformation motions. As such there is no need to solve separately generalized coordinates denoting the flexible motion from a reference set of coordinates denoting the rigid motion. In addition, as the nodal coordinates of the beam components are defined in the same kinematic manner as the physical coordinates of the rigid body components, no distinction need be made between the treatment of the flexible and rigid components of the multibody system other than the calculation of the internal force of the flexible member. Therefore, the salient feature of this type of formulation is that numerical solution procedures for the integration of spatial kinematic systems can be directly applied to the generalized coordinates of both the rigid and flexible components.

A multibody dynamics solution procedure, originally demonstrated on rigid body sys-

tems in previous studies ${ }^{38-41}$, is adopted for the above flexible multibody system equations 
of motion. The key to the procedure is a staggered implementation of the separate generalized coordinate integrator and constraint force solver modules. An improved variation of the explicit central difference algorithm, described in Section 4.1, is used to integrate the translational displacements and the angular velocity of the system. An algorithm based on the Euler parameter representation of finite rotations, described in Section 4.2 , is used to update the configuration orientation from the angular velocity. The computations of the Lagrange multipliers are then carried out in a separate routine, described in Section 4.3, which implicitly integrates a stabilized companion differential equation for the constraint forces in time.

\subsection{Explicit Generalized Coordinate Integrator}

The central difference explicit integration algorithm is written as

$$
\begin{aligned}
\dot{d}^{n+\frac{1}{2}} & =\dot{d}^{n-\frac{1}{2}}+h \ddot{d}^{n} \\
d^{n+1} & =d^{n}+h \dot{d}^{n+\frac{1}{2}} \\
\ddot{d}^{n+1} & =M^{-1} Q\left(d^{n+1}, \dot{d}^{n+1}\right)
\end{aligned}
$$

where the superscript $n=1,2,3, \cdots$ designates the discrete time station $t^{n}=n h$ and $h$ is the stepsize. Unlike in conventional structural dynamics, a straightforward application of (4.1) on the rotational equations

$$
J \dot{\omega}+\tilde{\omega} J \omega=f_{\omega}
$$

inherent in the multibody system equations of motion (3.32) leads to computational difficulties. In order to compute $\dot{\omega}^{n+1}$, it is necessary to have $\omega^{n+1}$. However, due to the inherent nature of the algorithm, only $\omega^{n+\frac{1}{2}}$ is available. It was shown ${ }^{41}$ that the naive approximation

$$
\omega^{n+1} \simeq \omega^{n+\frac{1}{2}}
$$

results in a computationally unstable integration of the angular velocity $\omega$. To correct this within the context of explicit computational sequences, an interlaced application of the central difference algorithm such that the displacements and velocities are advanced one-half time step at a time was proposed ${ }^{40,41}$. The algorithm advances the solution to the time station $t^{n+\frac{1}{2}}$ given the solutions of the two preceding time stations $t^{n-\frac{1}{2}}$ and $t^{n}$ as follows: 


$$
\begin{aligned}
& u^{n+\frac{1}{2}}=u^{n-\frac{1}{2}}+h \dot{u}^{n} \\
& \dot{u}^{n+\frac{1}{2}}=\dot{u}^{n-\frac{1}{2}}+h \ddot{u}^{n} \\
& \omega^{n+\frac{1}{2}}=\omega^{n-\frac{1}{2}}+h \dot{\omega}^{n} \\
& q^{n+\frac{1}{2}}=q\left(\omega^{n+\frac{1}{2}}\right) \\
& S^{n+\frac{1}{2}}=S\left(u^{n+\frac{1}{2}}, q^{n+\frac{1}{2}}\right) \\
& D^{n+\frac{1}{2}}=D\left(\omega^{n+\frac{1}{2}}\right), f^{n+\frac{1}{2}}=f\left(t^{n+\frac{1}{2}}\right) \\
& Q^{n+\frac{1}{2}}=Q\left(f^{n+\frac{1}{2}}, S^{n+\frac{1}{2}}, D^{n+\frac{1}{2}}\right) \\
& \lambda^{n+\frac{1}{2}}=\lambda\left(\lambda^{n}, Q^{n+\frac{1}{2}}\right) \\
& \ddot{u}^{n+\frac{1}{2}}=M^{-1}\left(Q_{u}^{n+\frac{1}{2}}-B_{u}^{T} \lambda^{n+\frac{1}{2}}\right) \\
& \dot{\omega}^{n+\frac{1}{2}}=J^{-1}\left(Q_{\omega}^{n+\frac{1}{2}}-B_{\omega}^{T} \lambda^{n+\frac{1}{2}}\right)
\end{aligned}
$$

The evaluation of the generalized rotational parameters $q$ to be obtained from the angular velocity, as represented by step $(d)$, will be detailed in Section 4.2 . The evaluation of the internal force $S$ from the current configuration coordinates $u$ and $q$, as represented by step (e), will be detailed in Section 5. The evaluation of the Lagrange multipliers $\lambda$, as represented by step $(\mathrm{h})$, will be detailed in Section 4.3. The algorithm proceeds to the next half time station $t^{n+1}$, now given the solutions at time stations $t^{n}$ and $t^{n+\frac{1}{2}}$, and thus the force and acceleration terms are evaluated twice each time step. The algorithm is initiated for time $t^{\frac{1}{2}}$ given initial conditions for time $t^{0}$ in the following manner:

$$
\begin{aligned}
& \text { (k) } \quad \dot{u}^{\frac{1}{2}}=\dot{u}^{0}+\frac{h}{2} \ddot{u}^{0} \\
& \text { (l) } \quad \omega^{\frac{1}{2}}=\omega^{0}+\frac{h}{2} \dot{\omega}^{0} \\
& \text { (m) } \quad \boldsymbol{u}^{1}=u^{0}+h \dot{u}^{\frac{1}{2}} \\
& \text { (n) } \quad u^{\frac{1}{2}}=\frac{1}{2}\left(u^{0}+u^{1}\right)
\end{aligned}
$$

from which steps $(d)$ through $(j)$ can be performed.

One last remark will be made on the angular velocity integration. The equations of motion were derived using body frame angular velocity components. The integration of these quantities shown in step $(c)$ is not formally correct as the components at different time steps are defined with respect to different body-fixed frames. This concern can be eliminated by applying the central difference update to the inertial components of the angular velocity. Step $(d)$ will then consist of an appropriate function of inertial angular velocity components. The integrated inertial angular velocities must be transformed to the moving reference frame before evaluating steps $(f)$ and $(j)$ since the equations of motion are written with respect to the body frame angular velocity description. The angular acceleration evaluated in step $(j)$ must then be transformed back to inertial reference frame before being integrated again in step $(c)$. 


\subsection{Rotational Parameter Integration}

The two-stage explicit integrator was applied to the translational displacement and velocity coordinates and the angular velocity coordinates. As the rotational orientation parameters are not directly integrable from the angular velocity vector, a procedure must be developed to update the configuration orientation given the angular velocity. Any finite rotation can be uniquely expressed by a rotation angle $\theta$ and an appropriate rotation axis n ${ }^{42}$. Two rotational parameterizations based on this description are the rotational vector $(\Theta)$ and the Euler parameters $\left(q_{0}, \mathbf{q}\right)$ defined respectively as

$$
\Theta=\theta \mathbf{n}, \quad\left\{\begin{array}{c}
q_{0} \\
\mathbf{q}
\end{array}\right\}=\left\{\begin{array}{r}
\cos \frac{\theta}{2} \\
\mathbf{n} \sin \frac{\theta}{2}
\end{array}\right\}
$$

The three parameters of the rotational vector are independent, while the four Euler parameters are subject to the constraints

$$
q_{0}^{2}+\mathbf{q}^{T} \mathbf{q}=1
$$

The rotation matrix is represented as a function of the Euler parameters as

$$
\mathbf{R}=\left[\begin{array}{lll}
2\left(q_{0}^{2}+q_{1}^{2}\right)-1 & 2\left(q_{1} q_{2}+q_{0} q_{3}\right) & 2\left(q_{1} q_{3}-q_{0} q_{2}\right) \\
2\left(q_{1} q_{2}-q_{0} q_{3}\right) & 2\left(q_{0}^{2}+q_{2}^{2}\right)-1 & 2\left(q_{2} q_{3}+q_{0} q_{1}\right) \\
2\left(q_{1} q_{3}+q_{0} q_{2}\right) & 2\left(q_{2} q_{3}-q_{0} q_{1}\right) & 2\left(q_{0}^{2}+q_{3}^{2}\right)-1
\end{array}\right] .
$$

The body frame components of the angular velocity tensor defined in (2.6) as

$$
\tilde{\omega}_{b}^{T}=\dot{\mathbf{R}} \mathbf{R}^{T}=\left[\begin{array}{ccc}
0 & \omega_{3} & -\omega_{2} \\
-\omega_{3} & 0 & \omega_{1} \\
\omega_{2} & -\omega_{1} & 0
\end{array}\right], \quad \omega_{b}=\left\{\begin{array}{l}
\omega_{1} \\
\omega_{2} \\
\omega_{3}
\end{array}\right\}_{b}
$$

has the Euler parameter representation ${ }^{42}$

$$
\left\{\begin{array}{c}
0 \\
\omega_{b}
\end{array}\right\}=2\left[\begin{array}{cc}
q_{0} & \mathbf{q}^{T} \\
-\mathbf{q} & q_{0} \mathbf{I}-\tilde{\mathbf{q}}
\end{array}\right]\left\{\begin{array}{c}
\dot{q}_{0} \\
\dot{\mathbf{q}}
\end{array}\right\}
$$

A similar expression for the inertial components of the angular velocity tensor

$$
\tilde{\omega}_{e}^{T}=\mathbf{R}^{T} \tilde{\omega}_{b}^{T} \mathbf{R}=\mathbf{R}^{T} \dot{\mathbf{R}}
$$

can be derived as

$$
\left\{\begin{array}{c}
0 \\
\omega_{e}
\end{array}\right\}=2\left[\begin{array}{cc}
q_{0} & \mathbf{q}^{T} \\
-\mathbf{q} & q_{0} \mathbf{I}+\tilde{\mathbf{q}}
\end{array}\right]\left\{\begin{array}{c}
\dot{q}_{0} \\
\dot{\mathbf{q}}
\end{array}\right\}
$$

The above definitions can be inverted to yield the expressions

$$
\left\{\begin{array}{c}
\dot{q}_{0} \\
\dot{\mathbf{q}}
\end{array}\right\}=\frac{1}{2}\left[\begin{array}{cc}
0 & -\omega_{b}^{T} \\
\omega_{b} & \tilde{\omega}_{b}^{T}
\end{array}\right]\left\{\begin{array}{c}
q_{0} \\
\mathbf{q}
\end{array}\right\}=\mathbf{A}_{b}\left(\omega_{b}\right)\left\{\begin{array}{c}
q_{0} \\
\mathbf{q}
\end{array}\right\}
$$


for the body frame components and

$$
\left\{\begin{array}{c}
\dot{q}_{0} \\
\dot{\mathbf{q}}
\end{array}\right\}=\frac{1}{2}\left[\begin{array}{cc}
0 & -\omega_{e}^{T} \\
\omega_{e} & \tilde{\omega}_{e}
\end{array}\right]\left\{\begin{array}{c}
q_{0} \\
\mathbf{q}
\end{array}\right\}=\mathbf{A}_{\mathbf{e}}\left(\omega_{e}\right)\left\{\begin{array}{c}
q_{0} \\
\mathbf{q}
\end{array}\right\}
$$

for the inertial frame components. A general representation

$$
\dot{q}=\mathbf{A}(\omega) q, \quad q=\left\{\begin{array}{c}
q_{0} \\
\mathbf{q}
\end{array}\right\}
$$

will be used to denote (4.9) or (4.10) given the angular velocity description. These inverse expressions are derived from (4.6) and (4.8) by incorporating the derivative of the constraint equation (4.4)

$$
\dot{q}_{0} q_{0}+\dot{\mathbf{q}}^{T} \mathbf{q}=0 \text {. }
$$

The configuration orientation is obtained from a numerical time discretization of the above Euler parameter - angular velocity representations. Among several possibilities, the approximation that satisfies the constraint condition (4.12) in the discrete sense is the following trapezoidal formula

$$
\frac{1}{h}\left(q^{n+1}-q^{n}\right)=\mathbf{A}\left(\omega^{n+\frac{1}{2}}\right) \frac{1}{2}\left(q^{n+1}+q^{n}\right) .
$$

Due to the structure of $\mathbf{A}$, the solution matrix can be analytically inverted such that the discrete orientation update

$$
q^{n+1}=\frac{1}{D}\left[I+\frac{h}{2} \mathbf{A}\left(\omega^{n+\frac{1}{2}}\right)\right]\left[I+\frac{h}{2} \mathbf{A}\left(\omega^{n+\frac{1}{2}}\right)\right] q^{n}
$$

where

$$
D=1+\frac{h^{2}}{4}\left(w_{1}^{2}+w_{2}^{2}+w_{3}^{2}\right)
$$

results. The final result is normalized to satisfy the constraint (4.4). The above equation is valid for either the body or inertial frame decomposition of the angular velocity as long as the corresponding form of $\mathbf{A}$ from (4.9) or (4.10) is used. The resulting update (4.14) involves only explicit computations and is readily incorporated into the two-stage explicit integration procedure.

\subsection{Constraint Force Solution Procedure}

A partitioned solution procedure has been employed to solve the generalized coordinates separately from the Lagrange multipliers. To effect a partitioned solution of the constraints, a stabilized companion differential equation for the constraint forces is formed by adopting the penalty procedure ${ }^{38,39}$. The penalty procedure uses the equations

$$
\lambda_{H}=\frac{1}{\epsilon} \Phi_{H}, \quad \dot{\lambda}_{N}=\frac{1}{\epsilon} \dot{\Phi}_{N}, \quad \epsilon \rightarrow 0
$$


as the basic constraint equations instead of (3.30) and (3.31) for the holonomic and the nonholonomic constraint conditions respectively. The penalty equations can be written in the general form, from (3.30) and (3.31), as

$$
\dot{\lambda}=\frac{1}{\epsilon} B \dot{z}, \quad \dot{z}=\left\{\begin{array}{c}
\dot{u} \\
\omega
\end{array}\right\}
$$

The numerical solution to the above companion differential equation is obtained as follows. The constrained equations of motion (3.32) are integrated once from (3.20) using the implicit integration rule

$$
\dot{z}^{n+\frac{1}{2}}=\dot{z}^{n}+\delta \ddot{z}^{n+\frac{1}{2}}, \quad \delta=\frac{h}{2}
$$

as

$$
\dot{z}^{n+\frac{1}{2}}=\delta M^{-1}\left(Q^{n+\frac{1}{2}}-B^{T} \lambda^{n+\frac{1}{2}}\right)+\dot{z}^{n} .
$$

This expression is substituted into (4.16) to obtain the stabilized differential equation for the Lagrange multipliers

$$
\epsilon \dot{\lambda}^{n+\frac{1}{2}}+\delta B M^{-1} B^{T} \lambda^{n+\frac{1}{2}}=\delta B M^{-1} Q^{n+\frac{1}{2}}+B \dot{z}^{n} .
$$

The same integration rule is applied to this equation to result in the discrete update

$$
\begin{gathered}
\left(\epsilon I+\delta^{2} B M^{-1} B^{T}\right) \lambda^{n+\frac{1}{2}}=\epsilon \lambda^{n}+r_{\lambda}^{n+\frac{1}{2}} \\
r_{\lambda}^{n+\frac{1}{2}}=\delta^{2} B M^{-1} Q^{n+\frac{1}{2}}+\delta B \dot{z}^{n} .
\end{gathered}
$$

The same procedure can also be derived with different integration rules. The update of the Lagrange multipliers, performed for each half time step, is easily adapted into the two-stage explicit integration procedure.

\section{Internal Force Computations}

The algorithmic treatment of the nonlinear stiffness operator is addressed in this section. The explicit generalized coordinate integrator of the previous section requires an evaluation of the internal force at a current time step $t^{n+1}$ from the coordinates of the beam configuration at that time. The internal force is first evaluated on the element level for all the finite elements comprising the flexible component from (3.26) as

$$
\left\{\begin{array}{c}
S_{I}^{e} \\
S_{I}^{b}
\end{array}\right\}^{E}=\left[B_{I}^{E}\right]^{T}\left\{\begin{array}{l}
N_{\gamma} \\
M_{\kappa}
\end{array}\right\}^{E}
$$

after which these individual element computations are assembled to form the internal force of the discrete beam. The necessary computations to be described are the evaluations of 
the discrete strain operator $\left[B_{I}^{E}\right]$ defined in (3.23) and the resultant stresses $N_{\gamma}$ and $M_{\kappa}$ respectively.

The Timoshenko beam formulation in which the translational degrees of freedom are independent from the rotational degrees of freedom requires an approximation within the element such that these variables will be continuous across the element boundaries. Thus a two node finite element representing a linear interpolation of the translational and rotational variables is a sufficient discretization of the beam. To avoid the locking phenomenon, the interpolation of the rotational degrees of freedom associated with the transverse shear strain is underintegrated. After incorporating these concepts into (3.27), the resulting expression for the discrete strain operator is given by

$$
\left[B_{I}^{E}\right]=\left[\begin{array}{cccc}
-\frac{1}{\ell} \mathbf{T} & \frac{1}{\ell} \mathbf{T} & \frac{1}{2} \tilde{i}_{1} \mathbf{S}_{1}^{T} & \frac{1}{2} \tilde{i}_{1} \mathbf{S}_{2}^{T} \\
0 & \mathbf{0} & \mathbf{S}_{1}^{T}\left(\frac{1}{2} \tilde{\kappa}_{S}-\frac{1}{\ell} \mathbf{I}\right) & \mathbf{S}_{2}^{T}\left(\frac{1}{2} \tilde{\kappa}_{S}+\frac{1}{\ell} \mathbf{I}\right)
\end{array}\right]
$$

which acts on the virtual displacements and rotations

$$
\left\{\begin{array}{cccc}
\delta u_{1} & \delta u_{2} & \delta \alpha_{1} & \delta \alpha_{2}
\end{array}\right\}^{T}
$$

where the subscripts refer to the element node number. The convected frame $T$ matrix, body frame curvature tensor $\tilde{\kappa}_{S}$, and element neutral-axis length $\ell$ are constant quantities over the element domain, while the relative cross-section deformation $\mathbf{S}$ matrices are nodal quantities. The computation of these terms from the nodal displacement and rotation coordinates of the current configuration are detailed in Section 5.1.

A stress update procedure of the form

$$
\left\{\begin{array}{l}
N_{\gamma} \\
M_{\kappa}
\end{array}\right\}^{n+1}=\left\{\begin{array}{l}
N_{\gamma} \\
M_{\kappa}
\end{array}\right\}^{n}+\Delta\left\{\begin{array}{l}
N_{\gamma} \\
M_{\kappa}
\end{array}\right\}
$$

is used to derive the resultant stresses of the current configuration at time $t^{n+1}$ from the resultant stresses of the past configuration at time $t^{n}$. The simple additive form of the procedure, which was derived from the numerical integration of a rate-type constitutive law, is due to the use of a convected frame stress and conjugate strain decomposition. The resultant stress increments $\Delta N_{\gamma}$ and $\Delta M_{\kappa}$ are obtained via

$$
\Delta N_{\gamma}=\left[\begin{array}{ccc}
E A & 0 & 0 \\
0 & G A & 0 \\
0 & 0 & G A
\end{array}\right] \Delta \gamma \quad, \quad \Delta M_{\kappa}=\left[\begin{array}{ccc}
G J & 0 & 0 \\
0 & E I_{2} & 0 \\
0 & 0 & E I_{3}
\end{array}\right] \Delta \kappa
$$

A set of strain increments $\Delta \gamma$ and $\Delta \kappa$, which denote the change from time $t^{n}$ to $t^{n+1}$, are defined as a finite analogy to the infinitesimal virtual strains $\delta \gamma$ and $\delta \kappa$ derived in Section 2. A specific computational procedure designed for use with this incremental interpretation 
of the continuum-based formulation such that the computed finite strain increments are invariant to arbitrary rigid body motions is discussed in Section 5.2.

\subsection{Computation of the Strain Operator}

The reference frames introduced in the formulation, namely the body frame $\mathbf{b}$ attached to the cross-section and the convected frame a tangent to the deformed neutral axis, are computed as follows. The Euler parameters representing the orientation of the beam crosssection at the finite element nodes are output from the generalized coordinate integrator at each time step. The rotation matrices $\mathbf{R}_{\boldsymbol{i}}$, representing the $\mathbf{b}$ reference frame at each element node, are thus computed directly from the Euler parameter representation of a rotation matrix (4.5). This matrix contains rotational information of both that due to the rigid motion of the convected reference frame and the transverse shear and torsional deformations of the cross-section relative to the convected frame.

The neutral axis of the finite element is defined as the straight line connecting the two element nodes, the tangent of which is trivial, and is directly calculated from the translational displacements output from the generalized coordinate integrator. Given this tangent $\mathbf{a}_{1}$, the $\mathbf{a}_{2}$ vector is defined as the cross product of $\mathbf{a}_{1}$ with the $\mathbf{b}_{3}$ axis of $\mathbf{R}_{1}$, and the remaining axis $\mathbf{a}_{3}$ defined to complete the right-hand coordinate system. The computed axis $\left\{\mathbf{a}_{1}, \mathbf{a}_{2}, \mathbf{a}_{3}\right\}$, as shown in Figure 2, define the rows of the $\mathbf{T}$ matrix. The rotation matrices $\mathbf{S}_{i}$, defined at each element node as the relative difference between the element convected frame and the nodal body frames, are thus

$$
\mathbf{S}_{i}=\mathbf{R}_{\mathbf{i}} \mathbf{T}^{T}, \quad i=1,2
$$

The procedure is an approximation applicable for moderate strains such that the $\mathbf{S}_{i}$ matri-

ces contain information solely due to transverse shear and torsional deformations ${ }^{43}$. The rotation matrices of the discrete strain operator (5.2) have thus been defined.

The body frame components of the curvature tensor $\tilde{\kappa}_{S}^{T}$ defined in (3.18) as

$$
\tilde{\kappa}_{S}^{T}=\frac{\partial^{a} \mathbf{S}}{\partial \xi} \mathbf{S}^{T}=\left[\begin{array}{ccc}
0 & \kappa_{3} & -\kappa_{2} \\
-\kappa_{3} & 0 & \kappa_{1} \\
\kappa_{2} & -\kappa_{1} & 0
\end{array}\right], \kappa=\left\{\begin{array}{l}
\kappa_{1} \\
\kappa_{2} \\
\kappa_{3}
\end{array}\right\}
$$

are equivalent to

$$
\tilde{\kappa}_{S}^{T}=\frac{\partial^{e} \mathbf{R}}{\partial \xi} \mathbf{R}^{T}
$$

as the convected frame $T$ matrix is defined to be constant along the element domain where the differentiation is performed. This definition is completely analogous to the angular velocity tensor defined in (2.6) and motivates the use of an Euler parameter representation of the curvature completely analogous to the Euler parameter representation of angular 
velocity (4.6) as a basis for the computation of the element curvature from the nodal rotational variables. The Euler parameter - curvature representation is

$$
\left\{\begin{array}{l}
0 \\
\kappa
\end{array}\right\}=2\left[\begin{array}{cc}
q_{0} & \mathbf{q}^{T} \\
-\mathbf{q} & q_{0} \mathbf{I}-\tilde{\mathbf{q}}
\end{array}\right]\left\{\begin{array}{l}
\frac{\partial q_{0}}{\partial \xi} \\
\frac{\partial \mathbf{q}}{\partial \xi}
\end{array}\right\}=\mathbf{E}\left(q_{a}\right) \frac{\partial q}{\partial \xi}
$$

subject to the constraints

$$
q_{0}^{2}+\mathbf{q}^{T} \mathbf{q}=1, \quad \frac{\partial q_{0}}{\partial \xi} q_{0}+{\frac{\partial \mathbf{q}^{T}}{\partial \xi}}^{T} \mathbf{q}=0 .
$$

An approximation to be used in (5.7) that satisfies the constraint conditions in the discrete sense

$$
\frac{\partial q}{\partial \xi}=\frac{1}{\ell}\left(q_{2}-q_{1}\right), \quad q_{a}=\frac{\frac{1}{2}\left(q_{1}+q_{2}\right)}{\left\|\frac{1}{2}\left(q_{1}+q_{2}\right)\right\|}
$$

is evaluated using the Euler parameters of the element nodes output from the generalized coordinate integrator. It will be shown that this discrete computation is invariant to rigid rotations contained in the total nodal Euler parameters.

The simple normalized average of the nodal Euler parameters has a physical interpretation. The Euler parameters $q_{a}$ correspond to an average orientation, in a geometric sense, of the two nodal cross-section orientations. This is demonstrated from the following example characterizing a state of constant curvature of a finite element shown in Figure 3. The orientation of the convected element frame is characterized by a rotation of an angle $\phi$ about an axis $\mathbf{n}_{a}$ from the inertial reference frame, and the relative nodal cross-section orientations are characterized by a rotation from the convected frame of angles $-\tau$ and $\tau$ about axis $\mathbf{n}_{b}$ for nodes 1 and 2 respectively. The Euler parameters designating the total cross section orientation of the two nodes due to these combined effects can be expressed as

$$
\begin{aligned}
& q_{1}=\left\{\begin{array}{c}
\cos \frac{\phi}{2} \cos \frac{\tau}{2}+\mathbf{n}_{a} \cdot \mathbf{n}_{b} \sin \frac{\phi}{2} \sin \frac{\tau}{2} \\
-\cos \frac{\phi}{2} \sin \frac{\tau}{2} \mathbf{n}_{b}+\cos \frac{\tau}{2} \sin \frac{\phi}{2} \mathbf{n}_{a}-\sin \frac{\tau}{2} \sin \frac{\phi}{2} \mathbf{n}_{a} \times \mathbf{n}_{b}
\end{array}\right\} \\
& q_{2}=\left\{\begin{array}{c}
\cos \frac{\phi}{2} \cos \frac{\tau}{2}-\mathbf{n}_{a} \cdot \mathbf{n}_{b} \sin \frac{\phi}{2} \sin \frac{\tau}{2} \\
\cos \frac{\phi}{2} \sin \frac{\tau}{2} \mathbf{n}_{b}+\cos \frac{\tau}{2} \sin \frac{\phi}{2} \mathbf{n}_{a}+\sin \frac{\tau}{2} \sin \frac{\phi}{2} \mathbf{n}_{a} \times \mathbf{n}_{b}
\end{array}\right\}
\end{aligned}
$$

which is obtained by applying the quaternion product rule $e^{44}$ to the Euler parameter definitions

$$
q_{r_{1}}=\left\{\begin{array}{c}
\cos \frac{\tau}{2} \\
-\sin \frac{\tau}{2} \mathbf{n}_{b}
\end{array}\right\} \quad, \quad q_{r_{2}}=\left\{\begin{array}{c}
\cos \frac{\tau}{2} \\
\sin \frac{\tau}{2} \mathbf{n}_{b}
\end{array}\right\} \quad, \quad q_{a}=\left\{\begin{array}{c}
\cos \frac{\phi}{2} \\
\sin \frac{\phi}{2} \mathbf{n}_{a}
\end{array}\right\}
$$

of the relative nodal orientations and the convected orientation respectively. The average of the two nodal Euler parameters (5.10) is

$$
\frac{1}{2}\left(q_{1}+q_{2}\right)=\left\{\begin{array}{c}
\cos \frac{\phi}{2} \cos \frac{\tau}{2} \\
\cos \frac{\tau}{2} \sin \frac{\phi}{2} \mathbf{n}_{a}
\end{array}\right\} .
$$


the norm of which is $\cos \frac{\tau}{2}$. When normalized, the above average is identical to the average orientation of the two nodes given by $q_{a}$. It can be shown that for this example the discretization (5.9) when substituted into (5.7) gives the finite element curvature computation

$$
\kappa=\frac{4}{\ell} \sin \frac{\tau}{2} \mathbf{n}_{b}
$$

which approximates the true curvature strain $\frac{2}{\ell} \tau \mathbf{n}_{b}$. The computation retains only the rotation parameters $\tau$ originally defined relative to the rigid body orientation, and is thus invariant to the rigid body motions. For instances when the validity of the approximation is challenged, an incremental curvature computation can be made as discussed in the next section, from which the total curvature is obtained from an appropriate update procedure.

\subsection{Computation of the Strain Increments}

The strain increments are defined from the virtual strains (3.12) by replacing the variational operator $\delta$ with an incremental operator $\hat{\Delta}$ as

$$
\hat{\Delta} \gamma=\mathbf{T} \frac{\partial \hat{\Delta} u}{\partial \xi}+\left\{\begin{array}{c}
0 \\
-\hat{\Delta} \beta_{3} \\
\hat{\Delta} \beta_{2}
\end{array}\right\}, \quad \hat{\Delta} \kappa=\frac{\partial \hat{\Delta} \beta}{\partial \xi}
$$

such that $\hat{\Delta} u$ and $\hat{\Delta} \beta$ are finite analogs of the infinitesimal displacements and rotations $\delta u$ and $\delta \beta$. For computation purposes, it becomes necessary to decompose the convected frame components of the virtual rotations of the of the cross-section $\delta \beta$ into a rotation due to rigid body motion $\delta \varphi$ and that due to deformations $\delta \tau$ as

$$
\delta \beta=\delta \tau_{a}+\delta \varphi
$$

This relation is derived by substituting the following definitions

$$
\begin{gathered}
\delta \tilde{\beta}^{T}=\mathbf{S}^{T} \delta \tilde{\alpha}^{T} \mathbf{S}, \quad \delta \tilde{\alpha}^{T}=\delta \mathbf{R} \mathbf{R}^{T} \\
\delta \tilde{\varphi}^{T}=\delta \mathbf{T T}^{T}, \quad \delta \tilde{\tau}^{T}=\delta \mathbf{S} \mathbf{S}^{T}, \quad \delta \tilde{\tau}_{a}^{T}=\mathbf{S}^{T} \delta \tilde{\tau}^{T} \mathbf{S}
\end{gathered}
$$

into the identity

$$
\mathbf{R}=\mathbf{S} \mathbf{T}, \quad \delta \mathbf{R}=\delta \mathbf{S} \mathbf{T}+\mathbf{S} \delta \mathbf{T} .
$$

It is noted that $\delta \alpha, \delta \varphi$, and $\delta \tau$ represent moving frame or spatial components referred to the defining reference frame, whereas $\delta \beta$ and $\delta \tau_{a}$ represent material components referred back to the convected frame. From these definitions, the incremental strain $\hat{\Delta} \gamma$ is given by

$$
\hat{\Delta} \gamma=\mathbf{T} \frac{\partial \hat{\Delta} u}{\partial \xi}+\left\{\begin{array}{c}
0 \\
-\hat{\Delta} \varphi_{3} \\
\hat{\Delta} \varphi_{2}
\end{array}\right\}+\left\{\begin{array}{c}
0 \\
-\hat{\Delta} \tau_{a_{3}} \\
\hat{\Delta} \tau_{a_{2}}
\end{array}\right\}
$$


representing the membrane strain and transverse shear strain increments. Likewise, the incremental curvature representing the torsion and bending strains is given by

$$
\hat{\Delta} \kappa=\frac{\partial \hat{\Delta} \tau_{a}}{\partial \xi}
$$

as the incremental rotations $\Delta \varphi$ defined from the $\mathbf{T}$ matrix are constant over the element length.

Essential for the use of these incremental strains is a proper definition and subsequent computation of the finite displacement and finite rotation increments. The incremental translations are defined by

$$
\hat{\Delta} u \equiv u^{n+1}-u^{n}
$$

as the displacements are true vector quantities. The incremental rotations are defined as follows. Rotations are updated by the product of orthogonal matrices via either ${ }^{24}$

$$
\begin{aligned}
\mathbf{R}^{n+1} & =\mathbf{R}_{(l)} \mathbf{R}^{n}=e^{\tilde{\theta}^{T}} \mathbf{R}^{n} \\
& =\mathbf{R}^{n} \mathbf{R}_{(r)}=\mathbf{R}^{n} e^{\bar{\theta}^{T}}
\end{aligned}
$$

using the rotational vectors $\theta$ or $\Theta$ based on the spatial or material reference frames respectively. It can be seen from the linearizations of the left and right rotational updates ${ }^{24}$

$$
\begin{gathered}
\mathbf{R}^{n+1} \simeq \mathbf{R}^{n}+\delta \mathbf{R} \\
\delta \mathbf{R}=\tilde{\theta}^{T} \mathbf{R}^{n}=\mathbf{R}^{n} \tilde{\Theta}^{T}
\end{gathered}
$$

that the virtual rotations

$$
\delta \tilde{\varphi}^{T}=\delta \mathbf{T} \mathbf{T}^{T}, \quad \delta \tilde{\tau}_{a}^{T}=\mathbf{S}^{T} \delta \mathbf{S}
$$

correspond to spatial and material rotation updates

$$
\mathrm{T}^{n+1}=\Delta \mathrm{T} \mathrm{T}^{n}, \quad \mathrm{~S}^{n+1}=\mathrm{S}^{n} \Delta \mathrm{S}
$$

respectively. Thus $\hat{\Delta} \varphi$ and $\hat{\Delta} \tau_{a}$ are defined as the rotational vectors parameterizing the matrices $\Delta \mathbf{T}$ and $\Delta \mathbf{S}$ respectively. Two different approximate methods which then extract this pseudovector from the given rotation matrix are used to obtain the incremental rotations. The particular approximation methods are chosen such that objective computations of the incremental strains (5.12) and (5.13) are achieved.

To this end, the first two terms of (5.12)

$$
\Delta \gamma_{1}=\mathbf{T} \frac{\partial \hat{\Delta} u}{\partial \xi}+\left\{\begin{array}{c}
0 \\
-\hat{\Delta} \varphi_{3} \\
\hat{\Delta} \varphi_{2}
\end{array}\right\}
$$


must be computed such that the $\hat{\Delta} \varphi$ rotation increment compensates for the rigid rotation contained in the displacement increment $\hat{\Delta} u$ defined in (5.14). To accomplish this, $\hat{\Delta} \varphi$ is computed by

$$
\hat{\Delta} \tilde{\varphi}^{T}=\Delta \mathbf{T}^{n+\frac{1}{2}}-\Delta \mathbf{T}^{n+\frac{1}{2}}{ }^{T}
$$

where $\Delta \mathbf{T}^{n+\frac{1}{2}}$ is defined from

$$
\mathbf{T}^{n+\frac{1}{2}} \equiv \exp \left(\frac{1}{2} \Delta \tilde{\varphi}^{T}\right) \mathbf{T}^{n} \equiv \Delta \mathrm{T}^{n+\frac{1}{2}} \mathbf{T}^{n} .
$$

The computation was derived from the linear approximation

$$
\mathbf{T}^{n+1} \simeq\left(I+\Delta \tilde{\varphi}^{T}\right) \mathbf{T}^{n}
$$

rewritten as

$$
\hat{\Delta} \tilde{\varphi}^{T}=\left(\mathbf{T}^{n+1}-\mathbf{T}^{n}\right) \mathbf{T}^{n+\frac{1}{2}}
$$

and introducing (5.19) to achieve a skew symmetric matrix. In order to preserve rigid motions, the matrix $\mathbf{T}$ in the first term of (5.17) must be evaluated as $\mathbf{T}^{n+\frac{1}{2}}$. This is shown as follows from an example of the rigid rotation of an element in which $\Delta \gamma_{1} \equiv 0$. From (5.20), it is seen that the rotational term of (5.17) becomes

$$
\left\{\begin{array}{c}
0 \\
-\hat{\Delta} \varphi_{3} \\
\hat{\Delta} \varphi_{2}
\end{array}\right\}=-\mathbf{T}^{n+\frac{1}{2}}\left(t_{\xi}^{n+1}-t_{\xi}^{n}\right), \quad t_{\xi}=\left\{\begin{array}{c}
T_{11} \\
T_{12} \\
T_{13}
\end{array}\right\}
$$

The finite element evaluation of the displacement term of (5.17) is given by

$$
\mathbf{T} \frac{\partial \hat{\Delta} u}{\partial \xi}=\mathbf{T} \frac{1}{\ell_{e}}\left(\hat{\Delta} u_{2}-\hat{\Delta} u_{1}\right)
$$

for the two-noded beam element of length $\ell_{e}$. For the rigid rotation of the second node about the first node, the incremental translational displacements are simply

$$
\hat{\Delta} u_{1}=0, \quad \hat{\Delta} u_{2}=\ell_{e}\left(t_{\xi}^{n+1}-t_{\xi}^{n}\right), \quad \frac{\partial \hat{\Delta} u}{\partial \xi}=t_{\xi}^{n+1}-t_{\xi}^{n} .
$$

as the direction cosines of the rotation are contained in the first row of the $\mathbf{T}$ matrix. Thus for $(5.17)$ to be identically equal to zero, it is necessary to evaluate (5.22) using $\mathrm{T}^{n+\frac{1}{2}}$. To obtain the true stretch with respect to the neutral-axis reference frame at the current configuration, we simply rotate the mid-configuration computation up to the current configuration as

$$
\Delta \mathrm{T}^{n+\frac{1}{2}}\left[\mathrm{~T}^{n+\frac{1}{2}} \frac{\partial \hat{\Delta} u}{\partial \xi}+\left\{\begin{array}{c}
0 \\
-\hat{\Delta} \varphi_{3} \\
\hat{\Delta} \varphi_{2}
\end{array}\right\}\right]
$$


As in the preceding analysis, the incremental displacements for an arbitrary rotation and stretch are given by

$$
\hat{\Delta} u_{1}=0, \quad \hat{\Delta} u_{2}=\left(\left(\ell_{e}+d\right) t_{\xi}^{n+1}-\ell_{e} t_{\xi}^{n}\right)
$$

where $d$ represents a stretch relative to the original element length $\ell_{e}$. The rotational expression (5.21) remains valid, and the bracketed term in (5.23) becomes

$$
\left(\frac{d}{\ell_{e}+d}\right) \mathbf{T}^{n+\frac{1}{2}} t_{\xi}^{n+1}
$$

Premultiplication of the above by $\Delta T^{n+\frac{1}{2}}$ results in the final computation

$$
\left(\frac{d}{\ell_{e}+d}\right) \mathbf{T}^{n+1} t_{\xi}^{n+1}=\left(\frac{d}{\ell_{e}+d}\right)\left\{\begin{array}{l}
1 \\
0 \\
0
\end{array}\right\}
$$

containing solely a measure of stretch regardless of the magnitude of the rigid rotation.

The incremental rotations $\hat{\Delta} \tau_{a}$ used to compute the remaining terms,

$$
\hat{\Delta} \gamma_{2}=\left\{\begin{array}{c}
0 \\
-\hat{\Delta} \tau_{a_{3}} \\
\hat{\Delta} \tau_{a_{2}}
\end{array}\right\} \quad \hat{\Delta} \kappa=\frac{\partial \hat{\Delta} \tau_{a}}{\partial \xi}
$$

representing transverse shear and curvature strains respectively, are computed independently from $\hat{\Delta} \varphi$ as follows. The rotation increments $\hat{\Delta} \tau_{a}$ are obtained from the matrix $\Delta S$ defined in (5.16) denoting the relative orientation between the current deformation matrix $\mathbf{S}^{n+1}$ and the past deformation matrix $\mathbf{S}^{n}$ rigidly rotated to the current convected frame. Another method to extract a rotation pseudovector from a given orthogonal rotation matrix given by ${ }^{43}$

$$
\hat{\Delta} \tilde{\tau}_{a i}^{T}=\frac{2\left(\Delta \mathbf{S}_{i}-\Delta \mathbf{S}_{i}^{T}\right)}{1+\operatorname{tr} \Delta \mathbf{S}_{i}}, \quad i=1,2
$$

is used to define $\hat{\Delta} \tau_{a}$ at each element node. The above method yields a simpler and more accurate computation of a rotation vector than (5.18). Whereas (5.18) was necessary to compute $\hat{\Delta} \varphi$ such that the rigid rotations within (5.17) are preserved, $(5.25)$ is used within (5.24) as this computation is made from matrices which by construction contain information solely due to deformation. Given the nodal rotation increments, the lochingfree form of the elemental shear strain is obtained from the nodal average as

$$
\hat{\Delta} \gamma_{2}=\frac{1}{2}\left\{\begin{array}{c}
0 \\
-\hat{\Delta} \tau_{a_{3}} \\
\hat{\Delta} \tau_{a_{2}}
\end{array}\right\}_{1}+\frac{1}{2}\left\{\begin{array}{c}
0 \\
-\hat{\Delta} \tau_{a_{3}} \\
\hat{\Delta} \tau_{a_{2}}
\end{array}\right\}_{2}
$$


and the elemental curvature is computed from the finite element approximation

$$
\hat{\Delta} \kappa=\frac{1}{\ell}\left(\hat{\Delta} \tau_{a_{2}}-\hat{\Delta} \tau_{a_{1}}\right)
$$

This completes the computational procedures for the incremental strains. The detailed strain computations of (5.12) and (5.13) are used in (5.4) to determine the stress increments, from which the current stress state is obtained from the update procedure (5.3).

\section{Numerical Examples}

The computational techniques, namely the staggered multibody dynamics solution procedure combining the generalized coordinate integrator and the constraint force solver discussed in Section 4 and the finite element computations of the beam internal force discussed in Section 5, have been implemented into a Fortran 77 software package. The result is a robust method which solves the present formulation of the equations of motion of an arbitrary assemblage of flexible beams and rigid bodies. In order to demonstrate the current software capabilities, the following examples highlighting the flexible motion of the beam component are presented.

The first example is included to verify the geometric stiffening phenomena exhibited by a rotating beam ${ }^{6,18,21,28}$. The beam is pinned at the left end; the other end remains free. The following material and geometric properties were used:

$$
\begin{gathered}
E A=2.8 \times 10^{7} \mathrm{lb}, \quad G A=1.0 \times 10^{7} \mathrm{lb}, \quad E I=1.4 \times 10^{4} \cdot \mathrm{lb}^{2} \\
\rho A=1.2 \mathrm{lbm} / \mathrm{in}, \quad \rho I=6.0 \times 10^{-4} \mathrm{lbm} \mathrm{in,} \quad l=10 \mathrm{in} .
\end{gathered}
$$

A prescribed angular rotation about the $\mathbf{e}_{3}$ axis of

$$
\theta(t)=\left\{\begin{array}{rl}
\frac{6}{15}\left[\frac{t^{2}}{2}+\frac{15}{2 \pi}^{2}\left(\cos \frac{2 \pi t}{15}-1\right)\right] \mathrm{rad} & 0 \leq t \leq 15 \mathrm{sec} \\
(6 t-45) \mathrm{rad} & t>15 \mathrm{sec}
\end{array}\right.
$$

is applied at the pinned end. The time history of the tip deflection relative to a reference frame coinciding with the prescribed angular position and the time history of several configurations of the beam are given in Figure 4. As alluded to in the introduction, an overall steady rotation of the beam gives rise to a centrifugal force which is responsible for a change in the bending stiffness that cannot be predicted using linear deformation theories. After initial increasing tip deflections, the beam begins to stiffen as the angular velocity increases due to the centrifugal inertia force. As the angular velocity reaches a constant state, the beam then reaches a steady state phase of small vibrations. This example shows the capability of the nonlinear strain formulation to automatically account for the geometric stiffening effect. The results are comparable to those presented by Simo 
and $\mathrm{Vu}-\mathrm{Quoc}{ }^{28}$. To reproduce these results with alternative methods as the substructuring technique ${ }^{21}$, a convergence analysis based on the selection of mode shapes must be performed.

The next examples exhibit the combined large deformation and large rotation capabilities of the present formulation. In the first instance, the beam is pinned as above and is subjected to given initial velocity impulses exciting various deformation mode shapes under planar motion. The following material and geometric properties were used in order to witness finite deformations:

$$
\begin{gathered}
E A=4.0 \times 10^{7} \mathrm{lb}, \quad G A=2.0 \times 10^{7} \mathrm{lb}, \quad E I=1.3 \times 10^{7} \mathrm{lb} \mathrm{in}^{2} \\
\rho A=.98 \mathrm{lbm} / \mathrm{in}, \quad \rho I=3.3 \times 10^{-2} \mathrm{lbm} \mathrm{in}, \quad l=200 \mathrm{in} .
\end{gathered}
$$

The initial velocity profiles with the resulting time histories of several deformed configurations are given in Figures 5, 6, and 7. It is noted the versatility of the formulation in its ability to capture the response to a variety of situations in which different fundamental modes of the beam are excited. The approach avoids the difficulty of tailoring the selection of modes shapes of the flexible components to the given problem at hand. The repeatability of the deformation shapes through time is due to the invariance of the internal force computations to the overall rigid motion. This property of computational objectivity is further illustrated in Figure 8 which shows the time history of the strain, kinetic, and total energy over four revolutions for the first bending mode example. The nature of the time integration and internal force algorithms are such that the conservation of energy is retained computationally, as seen by the fact that the total energy remains constant over all the revolutions. Similar results, not presented within, are obtained for the other deformation examples.

To present the applicability of the flexible beam component within the multibody dynamics framework, the final example of a spatial double pendulum is given. The double pendulum is modeled with two beams; a spherical joint connects the last node of the first beam to the first node of the second beam and also pins the first node of the first beam. It is noted that the joint connection can easily be accounted for from a finite element assemblage which leaves the rotational degrees of freedom free at the hinge location. The method was used to verify the results obtained using the Lagrange multiplier solver on the augmented equations described in Section 3.5. In the first case, the pendulum is subjected to a gravity field in the vertical z-direction and an initial velocity impulse in the horizontal $\mathrm{x}-\mathrm{y}$ plane such that soley rigid motion is excited. The problem is run for four cases of increasing beam flexibility as follows:

$\begin{array}{lll}\text { 1. } & E A=1.0 \times 10^{4} \mathrm{lb} & G A=0.5 \times 10^{4} \mathrm{lb} \\ 2 . & E A=1.0 \times 10^{3} \mathrm{lb} & G A=0.5 \times 10^{3} \mathrm{lb} \\ 3 . & E A=2.0 \times 10^{2} \mathrm{lb} & G A=1.0 \times 10^{2} \mathrm{lb} \\ \text { 4. } & E A=1.0 \times 10^{2} \mathrm{lb} & G A=0.5 \times 10^{2} \mathrm{lb}\end{array}$

with the remaining parameters 


$$
\rho A=1 \mathrm{lbm} / \text { in } \quad \rho I=.833 \times 10^{-3} \mathrm{lbm} \text { in } \quad l=1 \mathrm{in}
$$

held constant. The initial velocity impulse, and the spatial trajectories of the mass center of the second beam as projected on the $x-y$ and $x-z$ planes is given in Figure 9. The trajectory of the first case coincides exactly with a rigid body solution to the problem, and the slight deviation of the trajectories due to the increasing flexibility can be seen. The energy time histories for the problem, given in Figure 10, verify the computational objectivity of the algorithm as again energy is identically conserved. Again, the invariance of the internal force calculations in the three dimensional environment is witnessed by the negligible strain energy contribution for all of the flexible cases. The time integration of the spatial kinematics preserves the balance between the kinetic and potential energies of the problem. Next, the flexible double pendulum is given an initial velocity impulse to excite deformation motion as well as the rigid motion. For this case the parameters used were

$$
\begin{gathered}
E A=1.8 \times 10^{8} \mathrm{lb}, \quad G A=0.9 \times 10^{8} \mathrm{lb}, \quad E I=1.4 \times 10^{8} \mathrm{lb} \mathrm{in}^{2} \\
\rho A=.98 \mathrm{lbm} / \mathrm{in}, \quad \rho I=0.67 \mathrm{lbm} \mathrm{in}, \quad l=120 \mathrm{in} .
\end{gathered}
$$

The initial velocity profile, the resulting time histories of several deformed configurations and energy time history are given in Figure 11, exhibiting the large spatial rotation and deformation capabilities of the formulation. The energy conservation is retained for the computations of spatial deformations.

Further examples of large scale multibody systems are in process, and these results are to be presented in the near future.

\section{Concluding Remarks}

A flexible beam finite element that is readily incorporated into multibody dynamics applications has been presented. The beam formulation is based on fully nonlinear strain measures which remain invariant to rigid body motions. The model retains a Cauchy stress and physical strain description, and as such it can be easily interfaced with realtime slewing control applications as the measured strains can directly be used as a feedback signal without requiring sophisticated transformations. In addition, the formulation uses an inertial reference for the beam dynamics such that the degrees of freedom of the flexible component are defined in the same sense as the rigid components by including without distinction both the rigid and flexible deformation motions. The consequence is adaptability into multibody dynamics methodologies as numerical solution procedures for the integration of spatial kinematic systems can directly be applied to the generalized coordinates of both the rigid and flexible components. The success of the approach relies on an accurate. computation of the nonlinear internal force term. For this reason, the calculation of finite strain increments has been presented which are invariant to arbitrary rigid motions of the beam. The proposed methodology is suitable to treat the dynamics of flexible beams which 
undergo a variety of structural deformations in addition to the large overall motions. The same approach can be used in formulating other types of structural components.

\section{Acknowledgements}

The work reported herein was supported by NASA/Langley Research Center under Grant NAG-1-756. The authors wish to thank Dr. Jerry Housner for his interest and support during the course of the present work.

\section{References}

1. Ashley, H., "Observation on the dynamic behavior of flexible bodies in orbit," $A I A A$ J. 5 (1967), 460-469

2. Canavin, J.R. and Likins, P.W., "Floating reference frames for flexible spacecraft," $J$. of Spacecraft (1977), 724-732.

3. De Veubeke, B.F., "The dynamics of flexible bodies," Int J. Engng. Sci. 14 (1976), 895-913.

4. Grotte, P.B., J.C. McMunn, and R. Gluck, "Equations of motion of flexible spacecraft," J. of Spacecraft and Rockets 8 (1971), 561-567.

5. McDonough, T.B., "Formulation of the global equations of motion of a deformable body," AIAA J. 14 (1976), 656-660.

6. Kane, T. and Levinson, D., "Simulation of large motions of nonuniform beams in orbit: Part II - The unrestrained beam," J. Astronautical Sciences 29, (1981), 245-275.

7. Bodley, C., Devers, A., Park, A., and Frisch, H., "A digital computer program for dynamic interaction simulation of controls and structures (DISCOS)," NASA Technical Paper 1219, May 1978.

8. Song, J.O. and Haug, E.J., "Dynamic analysis of planar flexible mechanisms," Comp. Meth. Appl. Mech. Engrg. 24 (1980), 359-381.

9. Cavin, R.K. and Dusto, A.R., "Hamilton's principle: finite-element methods and flexible body dynamics," AIAA J. 15 (1977) 1684-1690.

10. Agrawal, O.P. and Shabana, A.A., "Dynamic analysis of multibody systems using component modes," Computers \& Structures 21 (1985), 1303-1312.

11. Agrawal, O.P. and Shabana, A.A., "Application of deformable-body mean axis to flexible multibody system dynamics," Comp. Meth. Appl. Mech. Engrg. 56 (1986), $217-245$.

12. Shabana, A.A. and Wehage, R.A., "A coordinate reduction technique for dynamic analysis of spatial substructures with large angular rotations," J. Struct. Mech. 11 (1983), 401-431. 
13. Yoo, W.S. and Haug, E.J., "Dynamics of articulated structures, Part I: Theory and Part II: Computer implementation and applications," J. of Structure Mechanics 14 (1986), 105-126 and 177-189.

14. Yoo, W.S. and Haug, E.J., "Dynamics of flexible mechanical systems using vibration and static correction modes," J. Mechanisms, Transmissions, and Automation in Design 108 (1986) 315-322.

15. Belytschko, T. and Hsieh, B.J., "Nonlinear transient finite element analysis with convected coordinates," Int. J. Num. Meth. Eng. 7 (1973), 255-271.

16. Belytschko, T., Schwer, L., and Klein, M.J., "Large displacement, transient analysis of space frames," Int. J. Num. Meth. Eng. 11 (1977), 65-84.

17. Housner, J., "Convected transient analysis for large space structures maneuver and deployment," Proc. the 25th Structures, Structural Dynamics and Materials Conference, AIAA Paper No. 84-1023, (1984) 616-629.

18. Housner, J.M., Wu, S.C., and Chang, C.W., "A finite element method for time varying geometry in multibody structures," Proc. the 29th Structures, Structural Dynamics and Materials Conference, April 1988, AIAA Paper No. 88-2234.

19. Laskin, R.A., Likins, P.W., and Longman, R.W., " Dynamical Equations of a FreeFree Beam Subject to Large Overall Motions," J. Astronautical Sciences 31 (1983), 507-528.

20. Kane, T.R., Ryan, R.R., and Banerjee, A.K., "Dynamics of a cantilever beam attached to a moving base," J. Guidance, Control, and Dynamics 10 (1987) 139-151.

21. Wu, S.C. and Haug, E.J., "Geometric nonlinear substructuring for dynamics of flexible mechanical systems," Int. J. Num. Meth. Engrg. 26 (1988) 2211-2226.

22. Bakr, E.M. and Shabana, A.A., "Geometrically nonlinear analysis of multibody systems," Computers \& Structures 23 (1986) 739-751.

23. Christensen, E.R. and Lee, S.W., "Nonlinear finite element modeling of the dynamics of unrestrained flexible structures," Computers \& Structures 23 (1986) 819-829.

24. Cardona, A. and Geradin, M., "A beam finite element nonlinear theory with finite rotations," Int. J. Num. Meth. Eng. 26 (1988), 2403-2438.

25. Iura, M. and Atluri, S.N., "On a consistent theory, and variational formulation of finitely stretched and rotated 3-D space-curved beams," Computational Mechanics (4) (1989), 73-88.

26. Simo, J.C., "A finite strain beam formulation. Part I: The three dimensional dynamic problem," Comp. Meth. Appl. Mech. Engrg. 49 (1985), 55-70.

27. Simo, J.C. and Vu-Quoc, L., "A three-dimensional finite strain rod model. Part II: Computational aspects," Comp. Meth. Appl. Mech. Engrg. 58 (1986), 79-116.

28. Simo, J.C. and Vu-Quoc, L., "Finite-strain rods undergoing large motions," Comp. Meth. Appl. Mech. Engrg. 66 (1988), 125-161. 
29. Antman, S.S., "Kirchhoff problem for nonlinearly elastic rods," Quat. J. Appl. Math $X X X I I ~ 3$ (1974), 221-240.

30. Reissner, E., "On a one-dimensional large-displacement finite-strain beam theory," Studies in Applied Mathematics 52 (1973), 87-95.

31. Reissner, E., "On finite deformations of space-curved beams," ZAMP 132 (1981), 734-744.

32. Wempner, G., Mechanics of Solids with Applications to Thin Bodies, Sijthoff \& Noordhoff, The Netherlands (1981).

33. Goldstein, H., Classical Mechanics, 2nd ed., Addison-Wesley (1980).

34. Malvern, L.E., Introduction to the Mechanics of a Continuous Medium, Prentice-Hall, Inc., Englewood Cliffs, N. J., (1969).

35. Eringen, A.C., Mechanics of Continua, Robert E. Krieger Publishing Co., Huntington, N.Y., (1980).

36. Zienkiewicz, O.C., 1977, The Finite Element Method, 3rd ed., McGraw Hill Book Company, Ltd, London.

37. Lanczos, L., The Variational Principles of Mechanics, 4th ed., University of Toronto Press, 1970.

38. Park, K.C., and Chiou, J.C., "Evaluation of constraint stabilization procedures for multibody dynamical systems," Proc. the 28th Structures, Structural Dynamics and Materials Conference, Part 2A, Monterey, CA, AIAA Paper No. 87-0927 (1987), 769-773.

39. Park, K.C., and Chiou, J.C., "Stabilization of computational procedures for constrained dynamical systems," Journal of Guidance, Control and Dynamics 11 (1988), 365-370.

40. Park, K.C., Chiou, J.C., and Downer, J.D., "A computational procedure for large rotational motions in multibody dynamics," Proc. the 29th Structures, Structural Dynamics and Materials Conference, Part 3, Williamsburg, VA, AIAA Paper No. 88-2416 (1988), 1593-1601 (also to appear in J. Guidance, Control and Dynamics).

41. Park, K.C., Chiou, J.C., and Downer, J.D., "An explicit-implicit staggered procedure for multibody dynamics analysis, Part I: Algorithmic aspects," Report No. CU-CSSC88-08, Center for Space Structures and Controls, University of Colorado (19S8).

42. Wittenburg, J., Dynamics of Systems of Rigid Bodies, B.G. Teubner, Stuttgart, 1977.

43. Rankin, C.C. and Brogran, F.A., "An element independent corotational procedure for the treatment of large rotations," J. of Pressure Vessel Technology 108 (19S6) $165-174$.

44. Geradin, M., Robert, G., and Buchet, P., "Kinematic and dynamic analysis of mechanisms: A finite element approach based on Euler parameters," in: Finite Element Methods for Nonlinear Problems(P. Bergan, ed.), Berlin, Heidelberg, New York, Springer, 1986. 


\section{FIGURES}

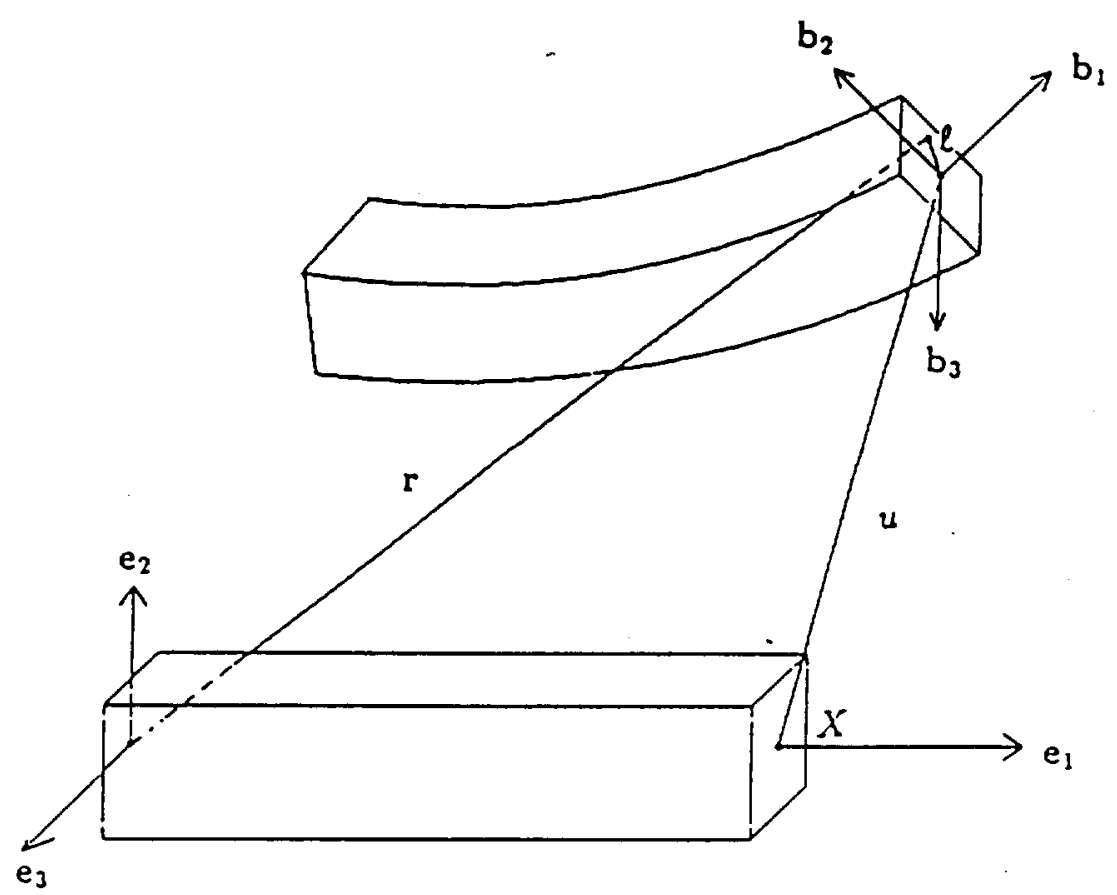

Figure 1. Beam Kinematics 


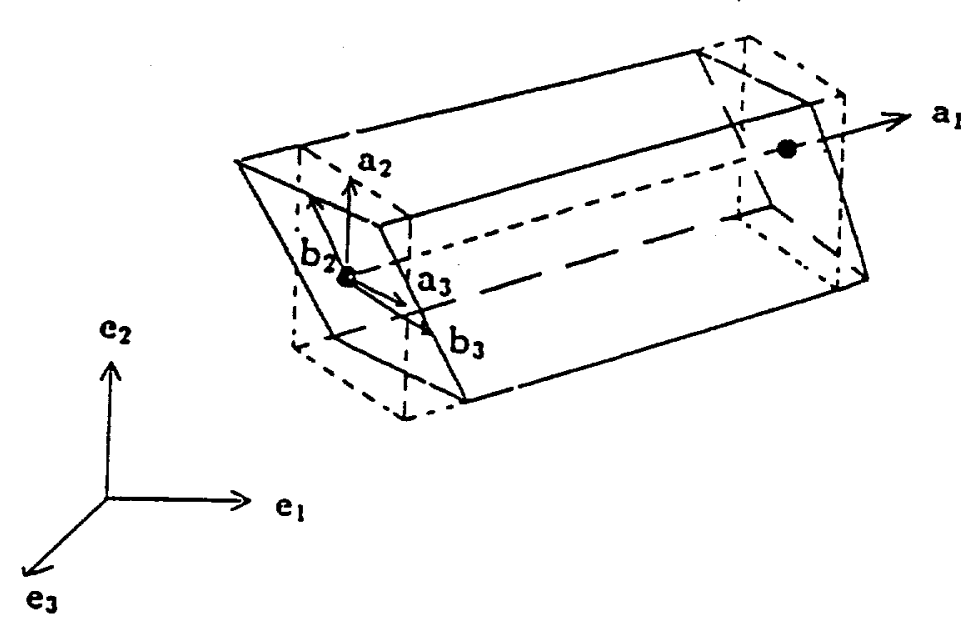

Figure 2. Convected Reference Frame

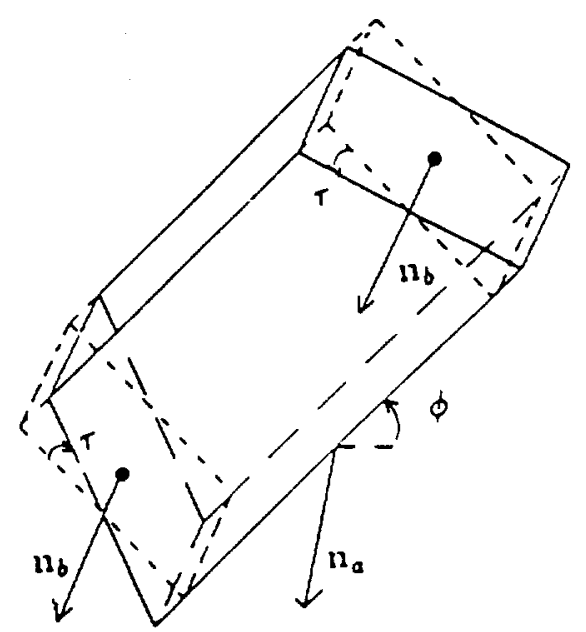

Figure 3. Pure Bending of Beam Element 

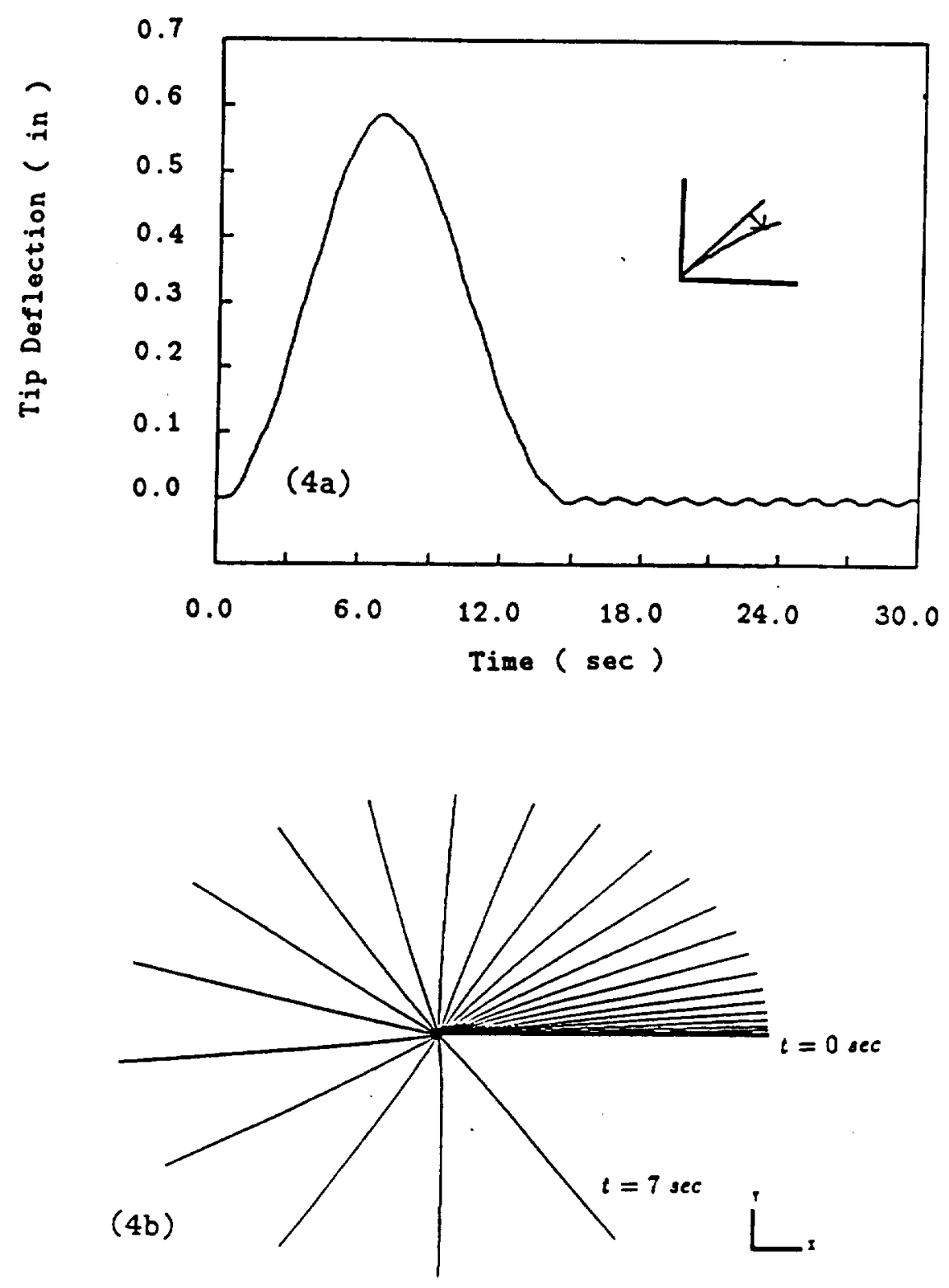

Figure 4. Geometric Stiffening ( 5 Elements):

(a) Tip Deflection vs. Time

(b) Displacement History 


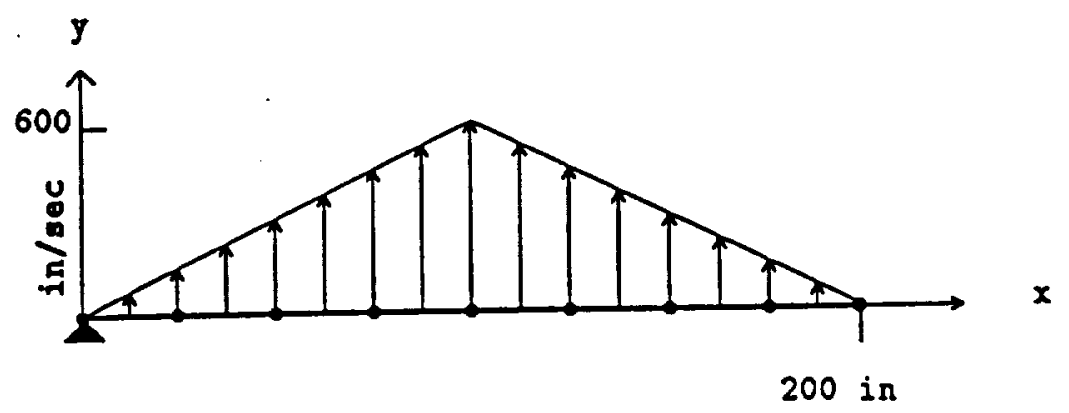

(5a)

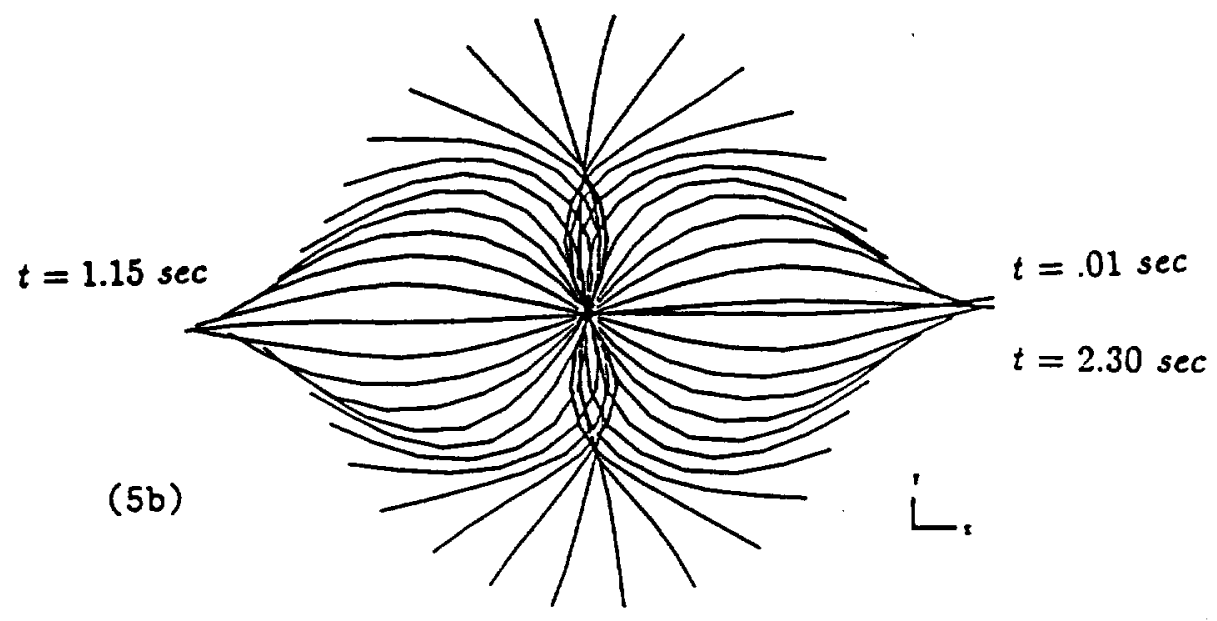

Figure 5. First Bending Mode ( 8 Elements ):

(a) Initial Beam Position vs. Initial Velocity Profile

(b) Displacement History 

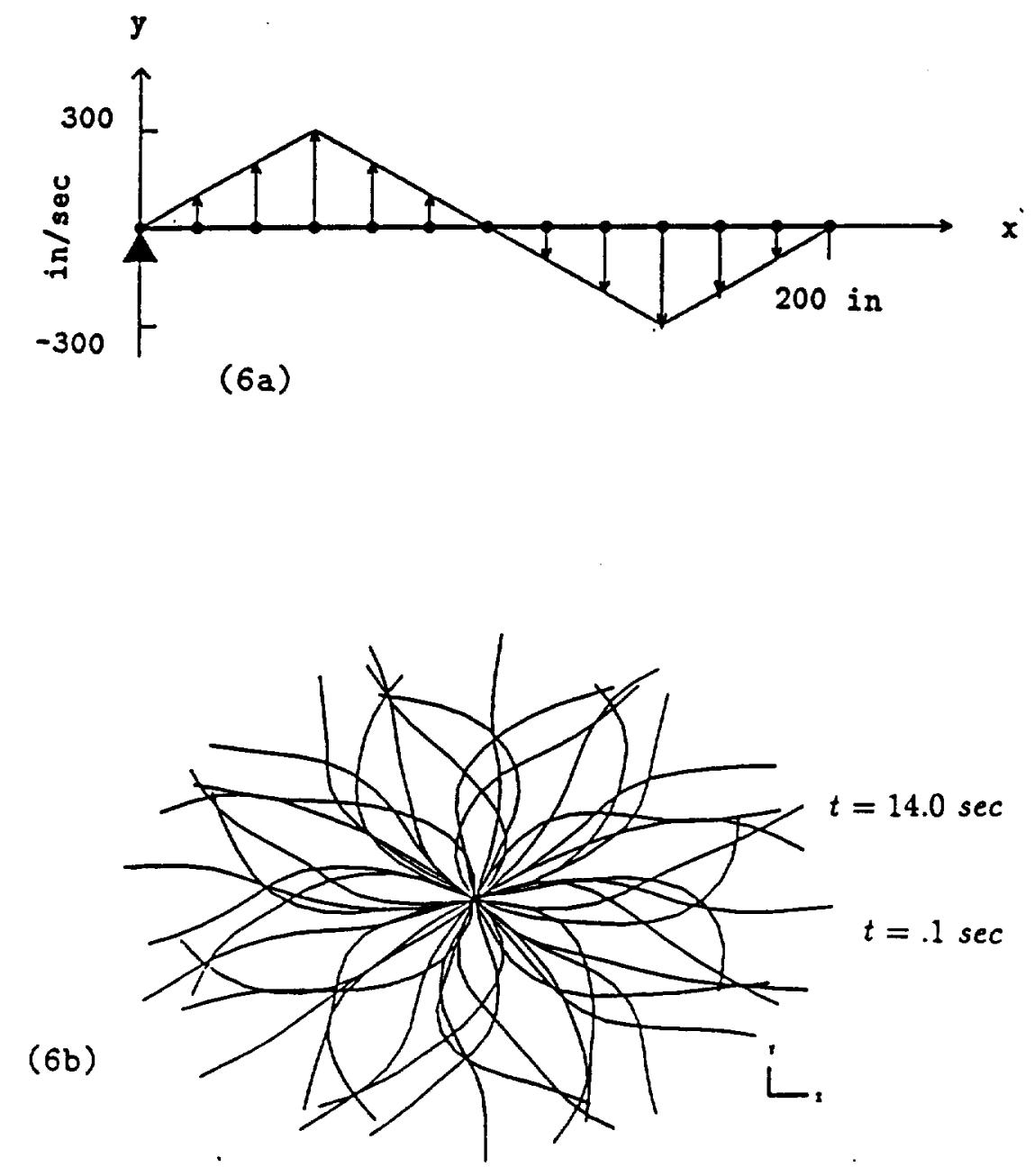

Figure 6. Second Bending Mode ( 12 Elements ):

(a) Initial Beam Position vs. Initial Velocity Profile

(b) Displacement History 

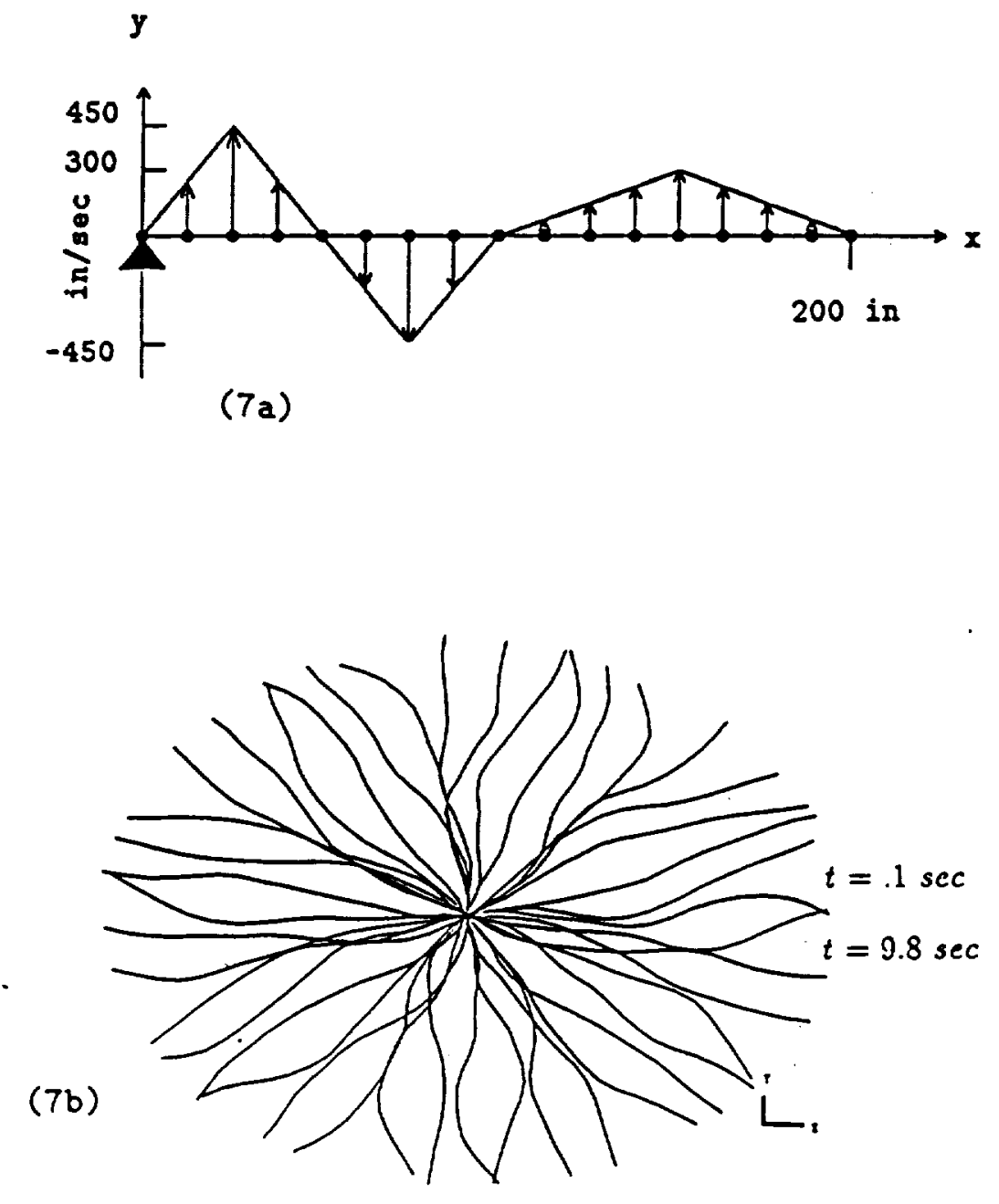

Figure 7. Combination Bending Mode ( 16 Elements ):

(a) Initial Beam Position vs. Initial Velocity Profile

(b) Displacement History 


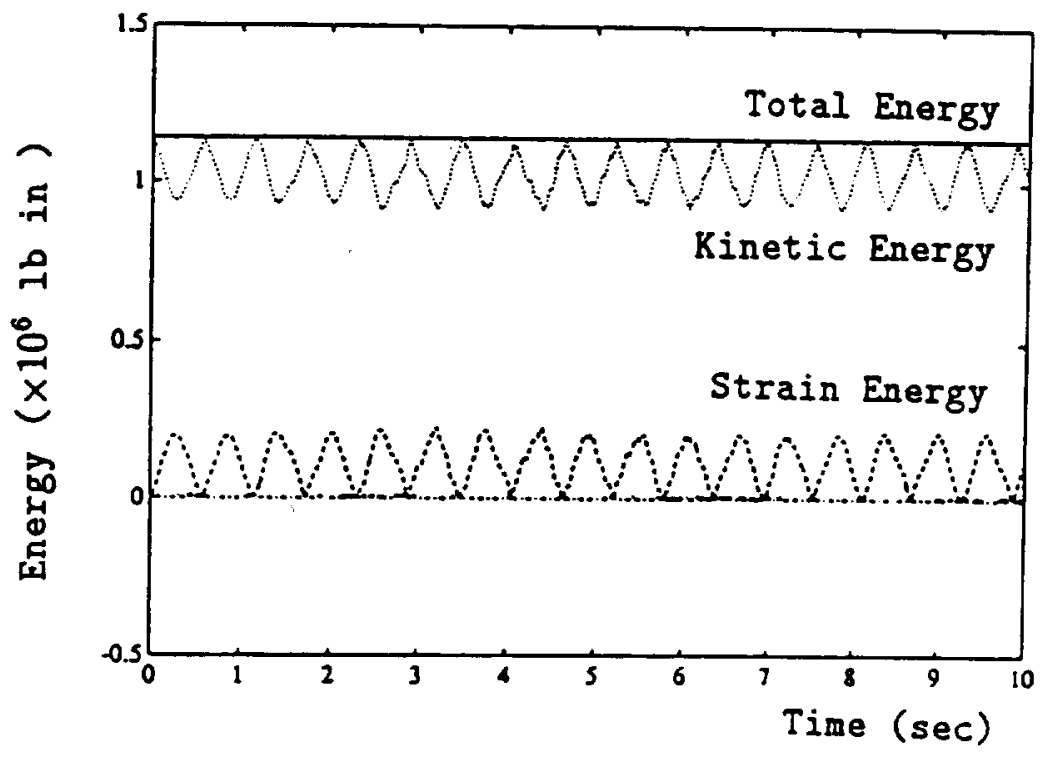

Figure 8. First Bending Mode: Energy Conservation 

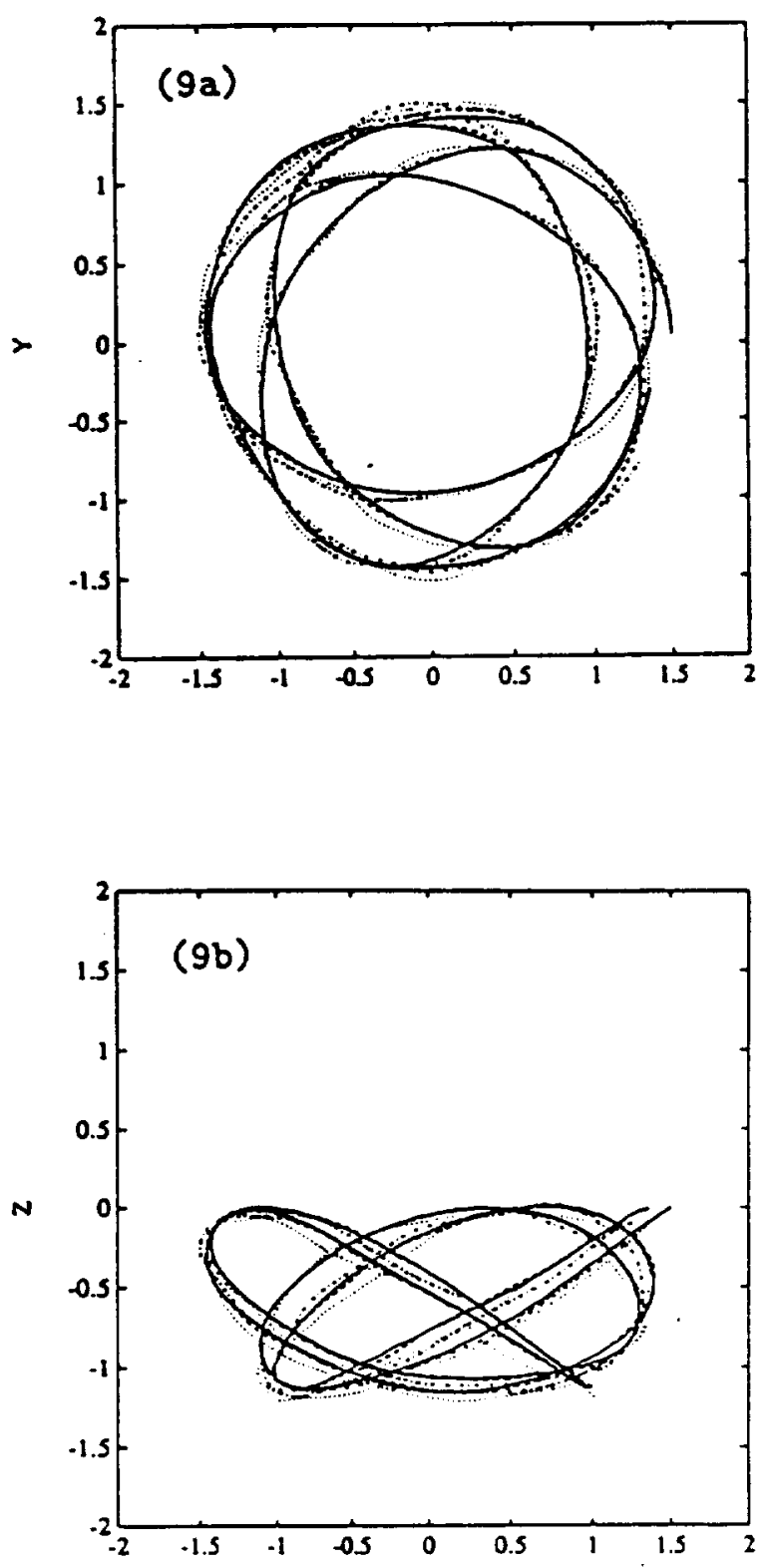

Figure 9. Spatial Double Pendulum ( 16 Elements ):

(a) Second Beam Trajectory: X-Y Plane

(b) Second Beam Trajectory: X $Z$ Z Plane 

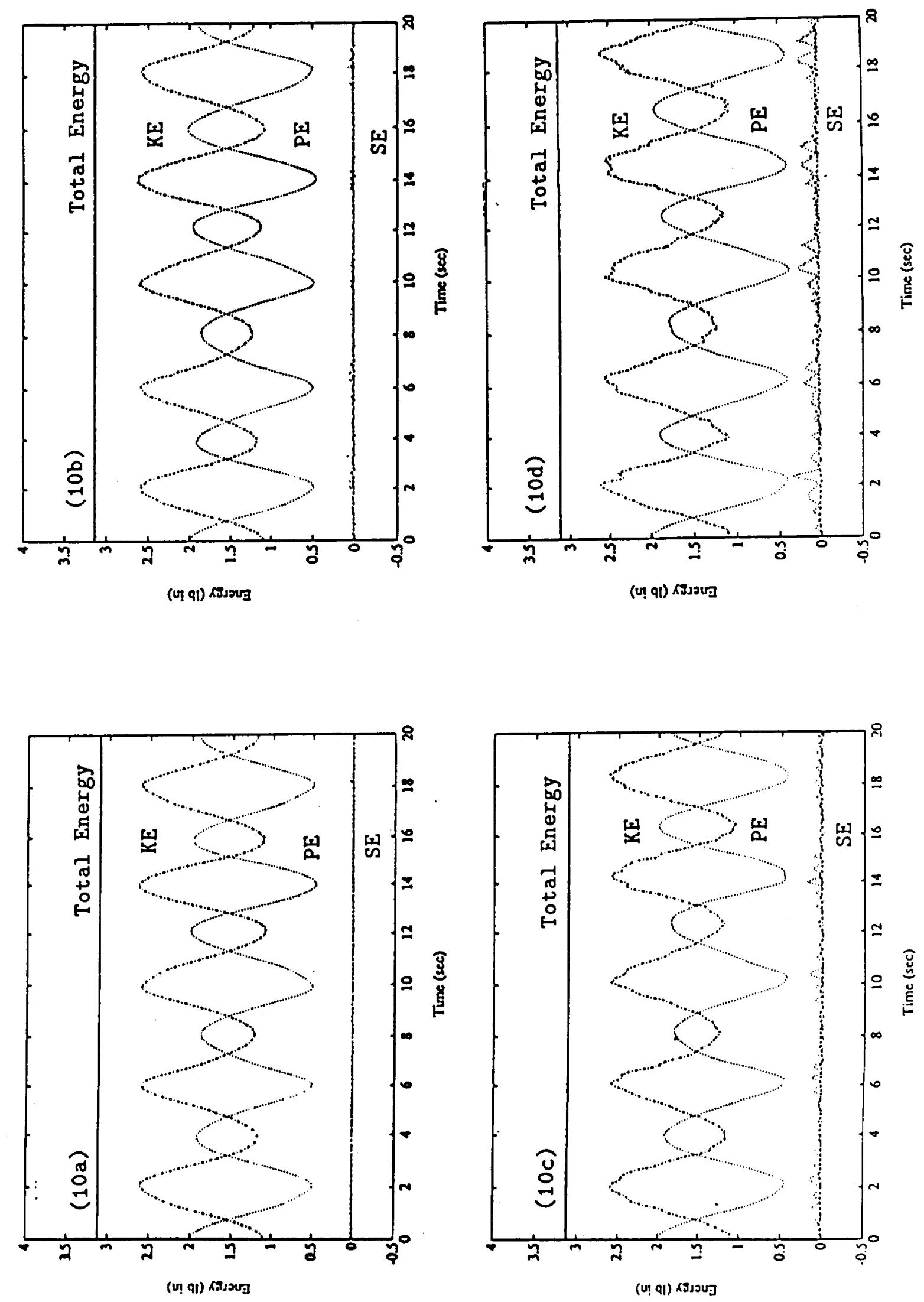

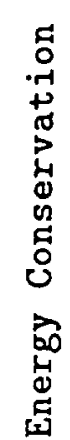

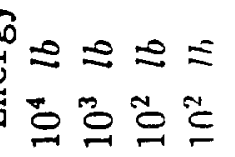

. $\times \times \times \times$

E

马ु

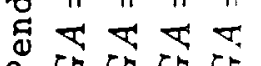

$0 \circlearrowright \circlearrowleft \circlearrowright \circlearrowright$

넘

$\approx \approx \approx=$

둥으음 등

$\rightarrow \times \times \times$



es || || ||

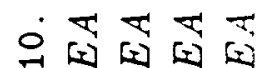

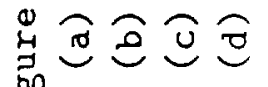
at 

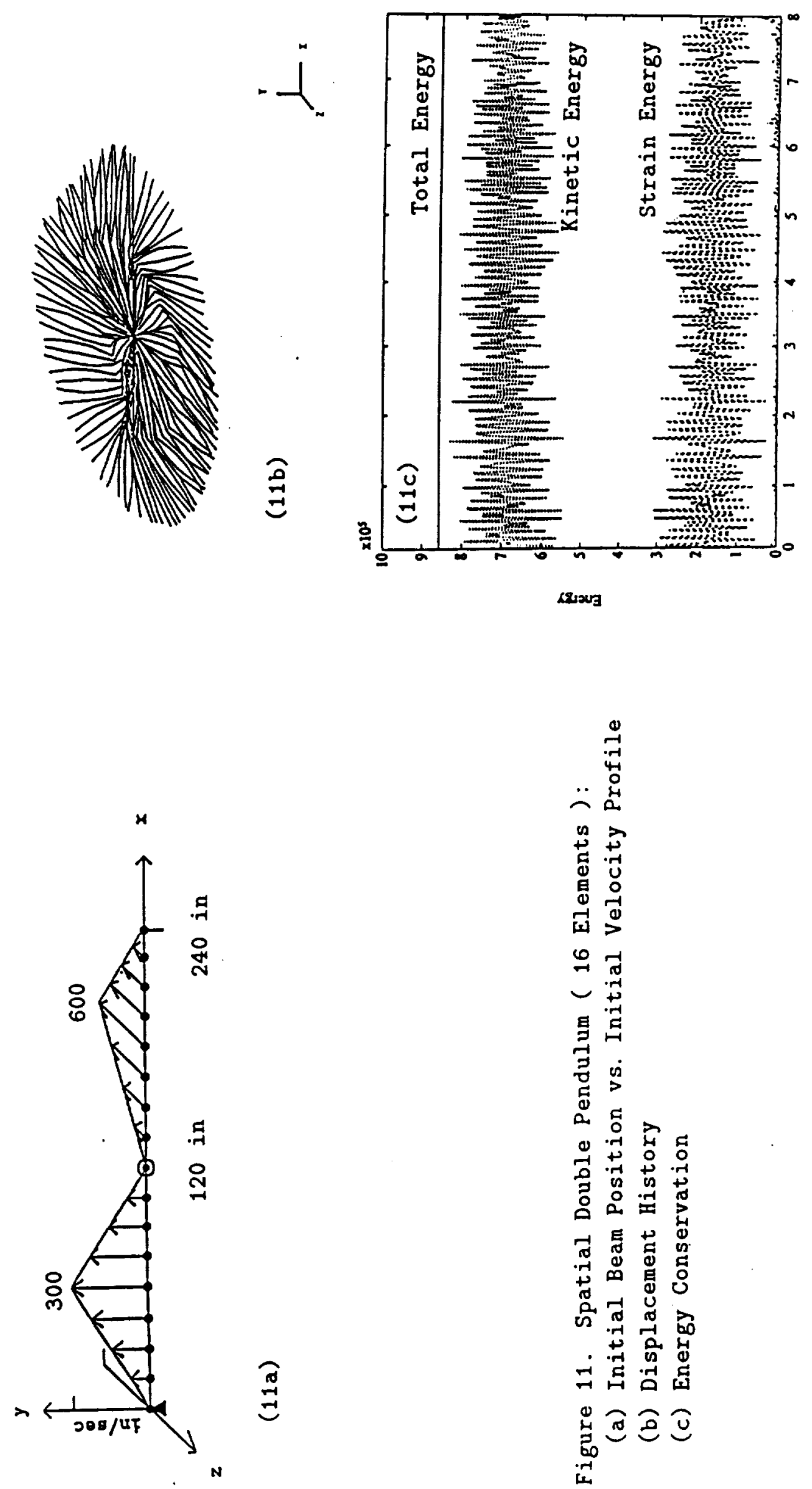